\title{
Numerical Spoiler Wake Investigations at the Borders of the Flight Envelope
}

\author{
Sven Geisbauer* \\ DLR Institute of Aerodynamics and Flow Technology, 38108 Braunschweig, Germany
}

\begin{abstract}
Modern CFD codes based on solving the Reynolds-averaged Navier-Stokes (RANS) equations are mostly applied to investigate the aerodynamic performance of an aircraft in its cruise design point or in high-lift configuration. For attached flows the prevailing tools have reached a high maturity for the linear regime of these flight conditions and are widely used in the aerodynamic design process. In contrast, the structural dimensioning of the aircraft based on the acting aerodynamic loads still relies on mostly semi-empirical methods or costly wind tunnel tests. In recent years an increasing effort has been undertaken to introduce high-fidelity CFD methods for aerodynamic loads prediction. Establishing current RANS methods in this area, especially in off-design conditions, is a challenging task, mainly due to the limitations of turbulence modeling, and requires their application within the entire flight envelope at guaranteed accuracy. The capabilities of these methods therefore need to be fostered for the complex flow cases encountered at the borders of the flight envelope.

A numerical feasibility study for a generic transport aircraft configuration with retracted and deployed spoilers is presented in this paper. In a first attempt, steady RANS methods using the one-equation Spalart-Allmaras, the two-equation Menter $k \omega$-SST and the SSG/LRR- $\omega$ Reynolds stress models have been applied to assess the suitability of the current approach in challenging flow conditions. The influence of the spoiler wake on the load distribution of the horizontal tail plane is investigated and a grid convergence study is carried out to verify the results. The influence of a grid adaptation to better capture the influence of the vortical flow of the spoiler wake is evaluated. In a next step, unsteady RANS (uRANS) computations were performed to capture the highly vortical, unsteady flow field behind the spoilers. The differences compared to the steady approach are assessed.
\end{abstract}

All investigations are performed with hexahedral-dominant grids, generated with the SOLAR software package, and the Reynolds-averaged Navier-Stokes solver TAU.

\section{Nomenclature}

$\begin{array}{llll}\alpha & \text { Angle of incidence } & C_{L} & \text { Lift coefficient } \\ \phi_{c / 4} & \text { Sweep at } 1 / 4 \text { chord line } & C_{m y} & \text { Pitching moment coefficient } \\ \eta & \text { Non-dimensional span coordinate } & C_{p} & \text { Pressure coefficient } \\ & (\eta=y / b / 2) & H & \text { Total layer thickness } \\ \lambda & \text { Wing taper ratio } & H_{t} & \text { Total enthalpy } \\ \Lambda & \text { Wing aspect ratio } & i_{H} & \text { HTP incidence angle } \\ \rho & \text { Density } & l & \text { Fuselage length } \\ b & \text { Span } & M & \text { Mach number } \\ c & \text { Chord length } & M_{\infty} & \text { Onflow Mach number } \\ c_{r e f} & \text { Wing mean chord length } & M_{M O} & \text { Maximum operating Mach number } \\ C_{D} & \text { Drag coefficient } & M_{D} & \text { Dive Mach number }\end{array}$

${ }^{*}$ Research Engineer, Transport Aircraft Department, AIAA member 


$\begin{array}{llll}n & \text { Load factor } & \text { CAD } & \text { Computer-aided design } \\ N & \text { Number of layers, number of grid points } & \text { CFD } & \text { Computational fluid dynamics } \\ P_{t} & \text { Total pressure } & \text { CS } & \text { (EASA) Certification specifications } \\ q & \text { Grid expansion ratio } & \text { dc } & \text { Drag count }\left[1 \mathrm{dc}=10^{-4}\right] \\ R e & \text { Reynolds number } & \text { DLR } & \text { German Aerospace Center } \\ S_{r e f} & \text { Reference area } & \text { EASA } & \text { European Aviation Safety Agency } \\ t & \text { Time } & \text { HTP } & \text { Horizontal tail plane } \\ t^{*} & \text { Characteristic time } & \text { lc } & \text { Lift count }\left[1 \text { lc }=10^{-3}\right] \\ \Delta t & \text { Unsteady time step size } & \text { RSM } & \text { Reynolds stress model } \\ v & \text { Velocity } & \text { SA } & \text { Spalart-Allmaras model } \\ v_{M O} & \text { Maximum operating air speed } & \text { SSG } / \text { LRR- } \omega & \text { Speziale-Sarkar-Gatski/Launder- } \\ v_{D} & \text { Dive speed } & & \text { Reece-Rodi Reynolds-stress model } \\ x_{r e f}, y_{r e f}, & \text { Moment reference coordinates } & \text { SST } & \text { Menter } k \omega \text {-SST model } \\ z_{r e f} & & \text { VTP } & \text { Vertical tail plane } \\ y^{+} & \text {Non-dimensional wall distance } & & \end{array}$

\section{Introduction}

\section{I.A. Background and Motivation}

Commonly used CFD methods like those based on solving the Reynolds-averaged Navier-Stokes (RANS) Uequations nowadays are a major source in the aerodynamic design process of an aircraft. For commercial transport aircraft RANS methods are intensely used to predict the aerodynamic performance in the cruise design point as well as in high-lift configuration. Especially for the former of these applications the quality of the results has reached high maturity. ${ }^{1,2}$ For attached flows the experience from the AIAA CFD Drag Prediction Workshop ${ }^{3}$ series has proven the ability of modern CFD methods to predict the differences between different configurations.

However, when regarding the entire flight envelope in which an aircraft must be able to operate safely these 'traditional' applications of RANS methods only represent a small part of it. Therefore, an increasing effort has been undertaken in recent years to extend the application of RANS methods towards the entire flight envelope. Reaching a similar maturity in those off-design cases with their challenging flow conditions, combined with complex aircraft configurations such as deployed spoilers, would allow RANS methods of playing a vital role in the process of aerodynamic loads prediction. So far, these loads have been provided by making use of semi-empirical methods or costly wind tunnel tests.

One project aiming at fostering modern CFD tools for aerodynamic loads prediction is ALEF ${ }^{\mathrm{a}}$, funded by the Seventh Framework Programme of the European Commission and involving twenty partners from the European aircraft industry and research establishments. The main objectives of ALEF are to extend the application range of modern CFD codes towards the borders of the flight envelope and, in a long-term view, to establish modern CFD codes as a major source for providing aerodynamic loads. To achieve this ambitious goal both high-fidelity RANS methods and variable-fidelity methods like those presented in Ref. 4 need to be applied.

\section{I.B. Spoiler Aerodynamics and Applications}

In the past, the research on spoiler control devices focussed on two-dimensional experimental and numerical studies. ${ }^{5,6,7}$ The transient aerodynamics during spoiler deployment and the influence of different turbulence models for calculating the unsteady, separated flow with compressible Navier-Stokes solvers was of vital interest. ${ }^{8,9}$ Others investigated the method of base-venting to reduce transient adverse spoiler effects. ${ }^{10}$

In recent years, the main focus shifted from a more or less isolated view on spoiler aerodynamics towards an assessment of the spoilers within the overall aircraft context. Both numerical and experimental studies were carried out to investigate spoiler aerodynamics with respect to handling qualities and loads prediction. ${ }^{11,12}$

${ }^{a}$ Aerodynamic Loads Prediction at Extremes of the Flight Envelope, 2009-2012. 
Most of these studies were based on structured grids, using one-equation or two-equation turbulence models in transonic flow. Latest studies validated both structured and unstructured RANS methods against an experimental database for a configuration with deployed spoilers. ${ }^{13}$ Both methods revealed promising results as long as the flow over the main wing remains attached.

Purely experimental studies assess the influence of the spoiler wake, focussing on the measurement and visualization of turbulences and vortices which might cause horizontal tail plane (HTP) buffet. ${ }^{14}$ Recently, an evaluation of spoilers under a more operational point of view has arisen. A sound investigation of a variation of the spoiler geometry and an assessment of multifunctional control devices with respect to the steep approach capabilities of a transport aircraft have been conducted. ${ }^{15,16}$

To summarize, the past studies on the three-dimensional assessment of spoiler aerodynamics focussed on RANS methods in combination with the so-called eddy viscosity turbulence models. Regarding the onflow conditions, the investigations were carried out at velocities not being higher than the cruise speed of modern airliners.

To the knowledge of the author, there is still a demand to assess the performance of an aircraft in using Reynolds stress models, especially when it comes to off-design flow conditions. Therefore, a computational study of a transport aircraft configuration with deployed spoilers at the borders of the flight envelope, using and comparing a Reynolds stress model with traditional eddy-viscosity turbulence models, has been carried out.

A common transport aircraft configuration with deployed spoilers ${ }^{\mathrm{b}}$, operating at high Mach number, was chosen to assess the feasibility of such computations using RANS methods. According to certification requirements the aircraft must sustain flight loads for all combinations of airspeed and load factor given within and at the borders of the so-called flight envelope (EASA CS 25.333). These borders are depicted in the v-n diagram of the aircraft. For large transport aircraft the sustainable load factor at cruise speed ranges from $n=-1$ to $n=2.5$. During normal operations the maximum operational airspeed $v_{M O} / M_{M O}$ shall not be exceeded deliberately (CS 25.1505). The flight control system of modern airliners provides aid to prevent the aircraft from exceeding this speed. However, to allow for a safety margin the aircraft is capable of flying at even higher speeds. The absolute speed limit is expressed in the V-n diagram by the so-called design dive speed $v_{D} / M_{D}$. Based on this airspeed the structure of the aircraft has to be designed such to sustain load factors ranging from $n=0$ to $n=2.5$ (CS 25.337). Exceeding this speed endangers the structural integrity of the aircraft. Modern wide-body airliners are typically designed for a maximum operating Mach number of $M_{M O} \approx 0.89$ and for a design dive Mach number of $M_{D} \approx 0.96$.

During abnormal operations near or at Mach dive speed spoilers are applied to enable higher sink rates at fixed air speed. These so-called drag devices need to be operable within the entire speed limit of the aircraft (CS 25.335f).

The massive occurrence of shock-boundary layer interaction on several aircraft components occuring in such flight conditions, combined with the highly vortical, unsteady flow field downstream of the spoilers still is a challenge for current RANS methods. In particular, the question in how far the vortical flow induced by the deployed spoilers affects the aerodynamic loads on the horizontal tail plane plays an important role in the sizing process of the aircraft. Information about aerodynamically induced vibrations in the empennage, the so called tail buffet, is difficult to predict at early design stages. To overcome this shortage, research establishments and aircraft manufacturers increasingly rely on unsteady RANS methods for investigating the buffet phenomenon in general, but also for estimating tail buffet loads. ${ }^{17,18,19}$

In the following an overview on the test cases, the onflow conditions and the mesh generation process will be given, followed by a brief presentation of the applied numerical methods. The steady results comprise the grid convergence behaviour, the influence of grid adaptation as well as the influence of the turbulence models on the spanwise load distributions of wing and HTP. Finally, the results of a uRANS simulation are compared with the steady approach.

\footnotetext{
b The term 'spoiler' used in this paper refers to their speed brake functionality only.
} 


\section{Test Case and Meshes}

\section{II.A. Test Case and Geometry}

The investigations are based on the DLR F12 configuration, a lightweight wind tunnel model of a modern transport aircraft configuration in cruise configuration. The geometry consists of fuselage, wing, horizontal and vertical (VTP) tail planes as depicted in figure 1. In recent years, this configuration has been extensively used for the measurement of unsteady dynamic derivatives. ${ }^{20}$ The DLR F12 configuration was chosen due to the fact that its geometric shape is designed for transonic flow conditions and, therefore, well-suited for the objectives of this work.

As the original DLR F12 configuration is lacking spoilers, a parametric spoiler model consisting of inboard and outboard spoilers was implemented into the CATIA V5 based CAD geometry, allowing for an easy variation of spoiler size, position and deployment angle. The latter parameters were derived from real aircraft data and scaled to fit the aircraft geometry in wind tunnel size. In contrast to real spoiler geometries the implemented model comprises the following geometric simplifications: the spoiler thickness is kept constant in all directions, inboard and outboard spoilers are modeled as one complete surface, i. e. no subdivision into segmented spoiler panels is considered and, thus, gap influences are neglected.

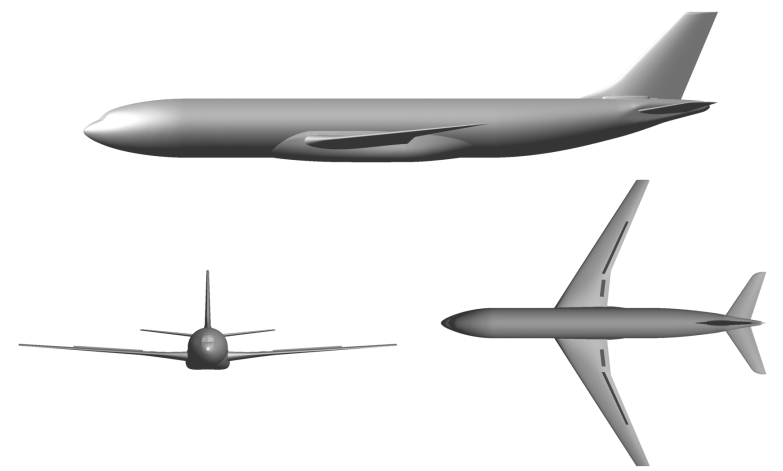

(a) Three-view drawing of the DLR F12 configuration with spoilers.

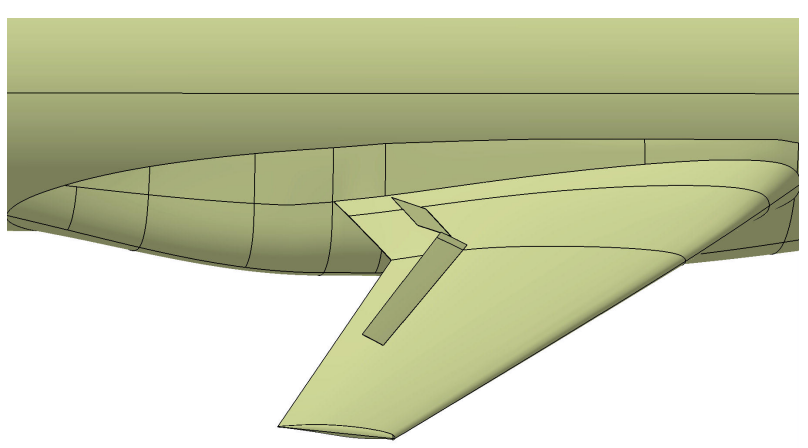

(b) Detailed view of the deployed inboard $\left(25^{\circ}\right)$ and outboard $\left(35^{\circ}\right)$ spoilers.

Figure 1. Overview of the DLR F12 configuration with deployed spoilers.

The computations were carried out for the clean-wing reference configuration and the configuration with deployed spoilers. For the latter the deployment angles were set to $25^{\circ}$ (inboard) and $35^{\circ}$ (outboard) and kept constant. The deployment angles were derived from the maximum speed brake setting of a real transport aircraft configuration.

Within the scope of computing flow conditions typically met at the borders of the flight envelope of commercial wide-body airliners, the DLR F12 model was scaled-up by a factor of $s \approx 29$. As listed in table 2 the scaling yields geometric dimensions very similar to those of common transport aircraft configurations and allows for an investigation at flight Reynolds numbers.

The RANS computations for both configurations were performed at $M_{\infty}=0.96, \alpha=0^{\circ}$ at an altitude of $35000 \mathrm{ft}$, leading to a Reynolds number of $R e=55 \cdot 10^{6}$ based on the mean aerodynamic chord. Due to the symmetric onflow conditions half-model computations were carried out to reduce the numerical effort. The incidence of the HTP was set to $i_{H}=0^{\circ}$ and remained unchanged, i. e. only untrimmed computations were considered. A variation of the load factor at Mach dive speed was accounted for in running a polar computation.

\section{II.B. Grid Generation}

The SOLAR grid generation software is applied to generate hybrid, quad-dominant surface and hexahedraldominant near-wall grids for the F12 configuration in clean-wing and in spoiler configuration. The software package was developed in a collaboration of BAE Systems, QinetiQ, Airbus and ARA. ${ }^{21,22}$

Compared to a discretization of surface and boundary layer based on triangles and prisms the application of quadrilateral and hexahedral elements allows for higher aspect ratios. For a given number of grid points the discretization error can be reduced due to a higher number of elements with an almost perpendicular alignment of their faces compared to the local onflow direction. 


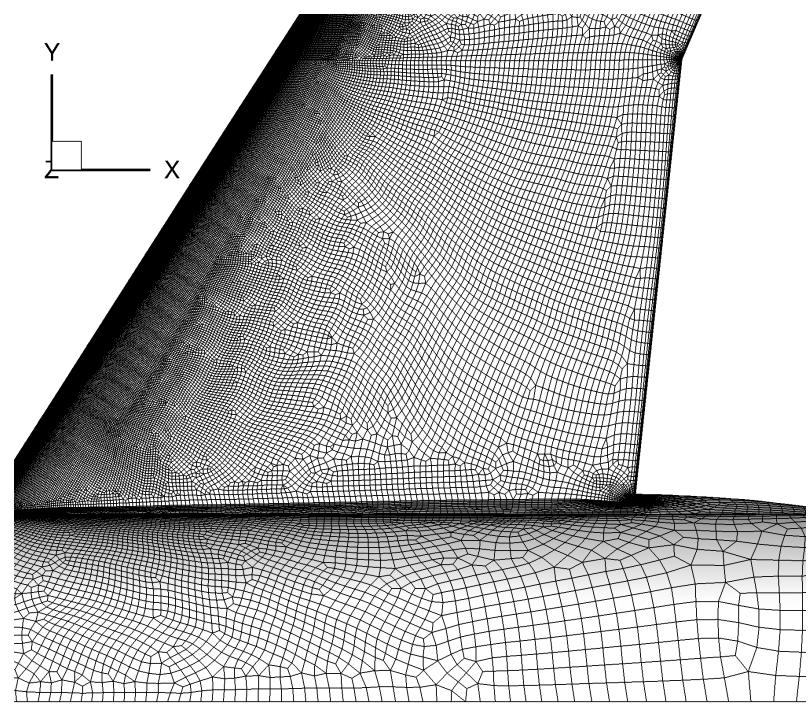

(a) Surface mesh resolution of the clean configuration.

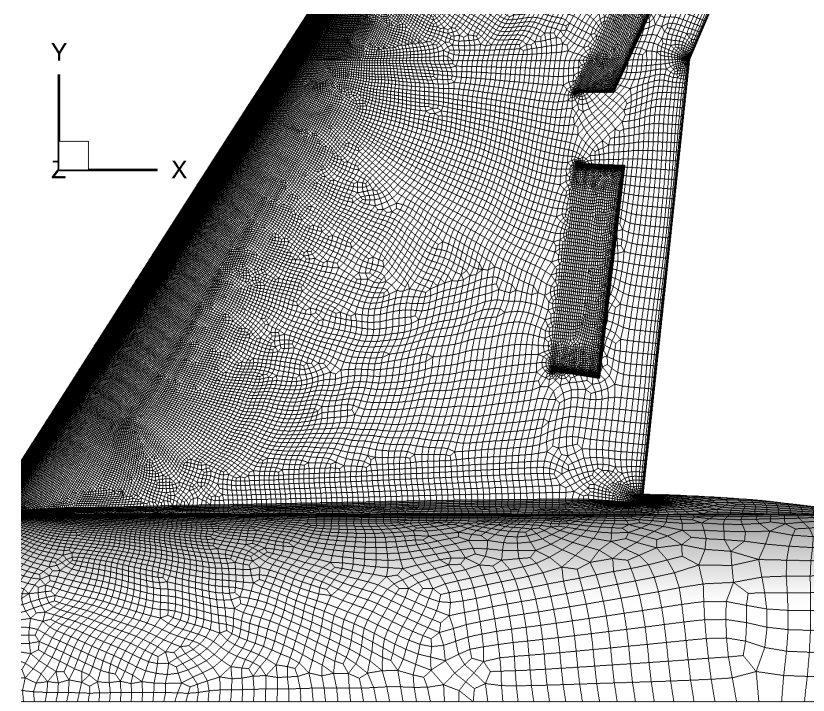

(b) Surface mesh resolution of the spoiler configuration.

Figure 2. Comparison of the surface meshes of the clean and spoiler configuration on the inboard section of the upper wing side.

In the framework of the fourth AIAA CFD Drag Prediction Workshop (DPW) the solution accuracy, the turbulence model influence and the grid convergence behaviour of two unstructured hybrid grid approaches, one of them being SOLAR, have been assessed for a similar configuration in combination with the DLR TAU-Code at transonic flow conditions. ${ }^{23}$

The sources, which control the element size in the mesh, were taken from the clean configuration mesh and have been reused during the mesh generation process of the spoiler configuration. Although the meshes for both configurations have been completely regenerated, this procedure allowed for largely identical surface meshes. To adequately resolve the spoiler geometry, additional sources have been applied. Figure 2 gives an overview on the surface mesh resolution near the wing-body intersection of both configurations. In both cases, the surface mesh discretization is largely identical. All meshes were generated such to yield a nondimensional wall distance of $y^{+} \leq 1$.

\section{II.B.1. Grid Convergence Study}

In order to assess the accuracy of the numerical solution a grid convergence study is applied according to the procedure presented in Ref. 24. As both the surface and volume mesh generation process is controlled by only one set of background sources, SOLAR allows for generating highly self-similar grid families by adjusting the spacing of these sources. This advantage was utilized to create three grid levels of varying size for the configuration with deployed spoilers. The total number of points between the grid levels differs by a factor of two, leading to a one-dimensional source scaling factor of $\sqrt[3]{2} \approx 1.2599$ for a three-dimensional grid.

Table 2. Dimensions of the scaled DLR F12 geometry.

\begin{tabular}{|l|c|c|}
\hline \hline Wing & $S_{\text {ref }}$ & $368.833 \mathrm{~m}^{2}$ \\
& $\mathrm{~b}$ & $58.672 \mathrm{~m}$ \\
& $c_{\text {ref }}$ & $7.28 \mathrm{~m}$ \\
& $\Lambda$ & 9.35 \\
& $\lambda$ & 0.3 \\
& $\phi_{c / 4}$ & $30^{\circ}$ \\
\hline Fuselage & $\mathrm{l}$ & $64.493 \mathrm{~m}$ \\
& $x_{r e f}$ & $29.970 \mathrm{~m}$ \\
& $y_{\text {ref }}$ & $0 \mathrm{~m}$ \\
& $z_{\text {ref }}$ & $-0.873 \mathrm{~m}$ \\
\hline HTP & $S_{\text {ref }}$ & $71.549 \mathrm{~m}^{2}$ \\
& $\mathrm{~b}$ & $21.325 \mathrm{~m}^{\circ}$ \\
& $\phi_{c / 4}$ & $30^{\circ}$ \\
\hline $\mathrm{VTP}$ & $S_{\text {ref }}$ & $55.524 \mathrm{~m}^{2}$ \\
& $\phi_{c / 4}$ & $40^{\circ}$ \\
\hline \hline
\end{tabular}

The first wall-normal spacing $a$ of the medium grid was chosen such to yield a $y^{+}=1$. With an user-defined expansion ration of $q=1.25,42$ wall-normal layers were generated with these settings. Under the constraint of keeping the total layer thickness $H$ constant, the coarse and fine grids were derived from the baseline medium grid. Table 3 gives an overview on the grid details of the spoiler configuration. Figure 3 gives an impression on the mesh resolution of center fuselage and upper wing for the different members of the grid family. 
Table 3. Overall grid details of the spoiler configuration after scaling.

\begin{tabular}{lccc}
\hline \hline Grid & coarse & medium & fine \\
\hline Surface points & $386 \cdot 10^{3}$ & $599 \cdot 10^{3}$ & $939 \cdot 10^{3}$ \\
No. of points in prism/hexa layer & $10.87 \cdot 10^{6}$ & $21.71 \cdot 10^{6}$ & $42.33 \cdot 10^{6}$ \\
No. of tetrahedral cells & $14.13 \cdot 10^{6}$ & $27.36 \cdot 10^{6}$ & $51.27 \cdot 10^{6}$ \\
Total number of cells & $24.84 \cdot 10^{6}$ & $48.80 \cdot 10^{6}$ & $93.13 \cdot 10^{6}$ \\
Total number of points (half model) & $12.43 \cdot 10^{6}$ & $24.94 \cdot 10^{6}$ & $48.61 \cdot 10^{6}$ \\
Total grid size ratio (based on medium grid) & $1 / 2$ & 1 & 1.95 \\
\hline First wall-normal layer spacing $(a)$ & $4.899 \cdot 10^{-3} \mathrm{~mm}$ & $3.887 \cdot 10^{-3} \mathrm{~mm}$ & $3.086 \cdot 10^{-3} \mathrm{~mm}$ \\
Expansion ratio $(q)$ & 1.3276 & 1.25 & 1.1928 \\
Max. no. of wall-normal layers $(N)$ & 34 & 42 & 52 \\
Total layer thickness $(H)$ & $183.0 \mathrm{~mm}$ & $182.7 \mathrm{~mm}$ & $153.3 \mathrm{~mm}$ \\
\hline \hline
\end{tabular}

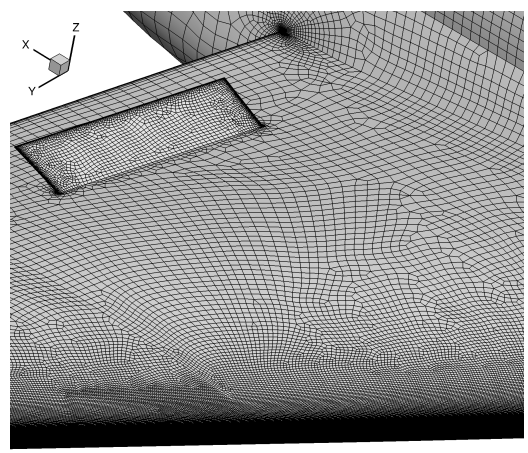

(a) Coarse grid resolution.

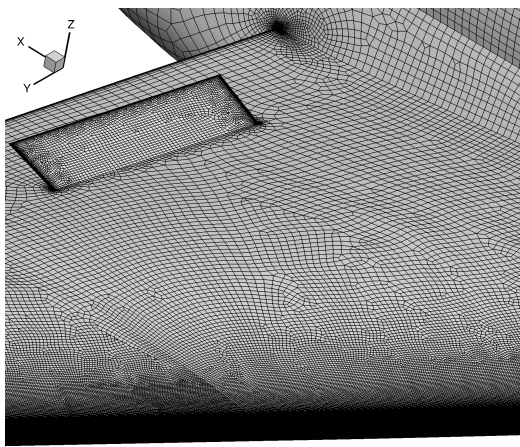

(b) Medium grid resolution.

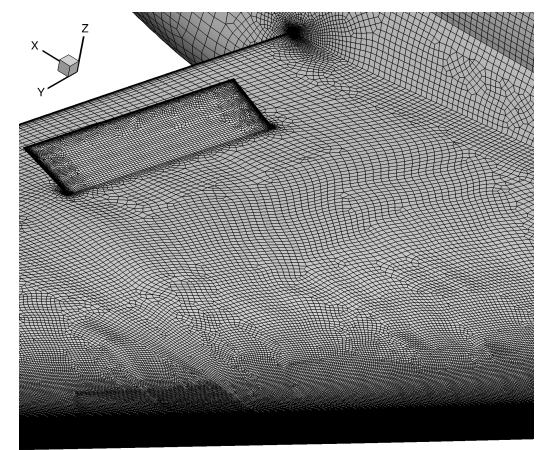

(c) Fine grid resolution.

Figure 3. Overview on the grid resolution on the upper side of the inboard wing section and inboard spoiler for the different members of the grid family.

\section{II.B.2. Grid Adaptation}

The automatic grid adaptation module of the DLR TAU-code ${ }^{25,26}$ was applied in order to better resolve the prevailing flow features like shocks and wake vortices. Based on the well-converged solution of the mediumsized spoiler grid, using the Spalart-Allmaras turbulence model, four grid refinement cycles were carried out. In the first adaptation cycle the entire computational domain was refined in regions of high gradients of total pressure, total enthalpy, density and velocity. The remaining adaptation cycles employed a special vortex detection sensor based on the $\lambda_{2}$-criterion. In contrast to the first cycle, the latter were carried out locally within a user-defined box in order to systematically refine the spoiler wake regions. The location and the dimensions of the box are depicted in figure 4 . The box extends from $32 \mathrm{~m}$ to $65 \mathrm{~m}$ in chordwise direction, from $3.5 \mathrm{~m}$ to $35 \mathrm{~m}$ in spanwise direction and from $-3 \mathrm{~m}$ to $4 \mathrm{~m}$ in $z$-direction. As shown the main sources for the generation of wake vortices, i. e. the spoilers and the wing tip, are located well within the adaptation region. Table 4 summarizes the grid adaptation process.

Table 4. Grid adaptation details for the spoiler configuration.

\begin{tabular}{lccc}
\hline \hline Adaptation cycle & $\begin{array}{c}\text { Refinement } \\
\text { parameter }\end{array}$ & $\begin{array}{c}\text { Total number of } \\
\text { grid points (half model) }\end{array}$ & $\begin{array}{c}\text { Relative increase } \\
\text { to former grid level }\end{array}$ \\
\hline Reference & - & $24.94 \cdot 10^{6}$ & - \\
Adaptation \#1 & $P_{t}, H_{t}, \rho, v$ & $27.73 \cdot 10^{6}$ & $+11 \%$ \\
Adaptation \#2 & $\lambda_{2}$ & $29.49 \cdot 10^{6}$ & $+6 \%$ \\
Adaptation \#3 & $\lambda_{2}$ & $31.51 \cdot 10^{6}$ & $+7 \%$ \\
Adaptation \#4 & $\lambda_{2}$ & $33.74 \cdot 10^{6}$ & $+7 \%$ \\
\hline \hline
\end{tabular}



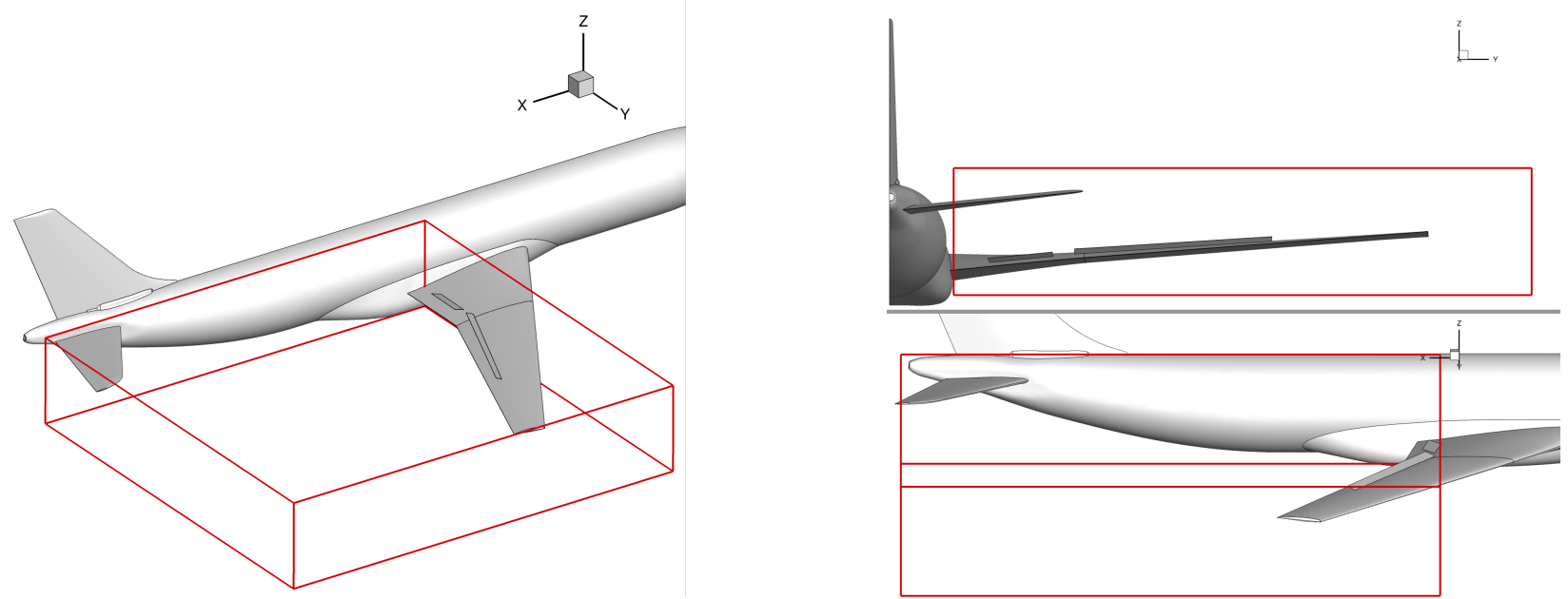

Figure 4. Location and size of the user-defined grid adaptation box.

\section{Numerical Flow Simulation}

The computations were performed with the DLR TAU-Code, a Reynolds-averaged Navier-Stokes solver which is under continuous development by DLR. ${ }^{27,28}$ The development of the TAU-code was substantially fostered within the German CFD project MEGAFLOW, consolidating the developments of DLR, aircraft industry and universities. TAU is an unstructured, edge-based solver in cell-vertex or cell-centered formulation, making use of the advantages of hybrid grids. The spatial discretization with either upwind or central schemes is based on a finite volume formulation. The temporal discretization may either be carried out with an explicit Runge-Kutta scheme or an implicit Lower-Upper Symmetric Gauss-Seidel (LU-SGS) scheme. Several convergence acceleration techniques like residual smoothing or multigrid schemes may be applied. Different turbulence models of varying fidelity have been implemented in TAU, of which three are used in this work.

The computations here were initialized at target onflow conditions, i. e. $M_{\infty}=0.96, \alpha=0^{\circ}$ and the atmospheric conditions equivalent to a flight at an altitude of $35000 \mathrm{ft}$. The computations have been started with an upwind scheme before switching to the Jameson-type central scheme ${ }^{29}$ until final convergence was reached. The temporal discretization was carried out with the implicit LU-SGS scheme.

Regarding the turbulence modeling, the one-equation Spalart-Allmaras ${ }^{30}$ (SA), the two-equation Menter $k \omega$-SST ${ }^{31}$ (SST) and the SSG/LRR- $\omega$ differential Reynolds stress model ${ }^{32}$ (RSM) have been applied. The two eddy-viscosity models may be considered as standard for aeronautical applications, especially when it comes to mainly attached flows. The RSM model implemented in TAU has revealed promising results within the AIAA CFD Drag Prediction Workshops III and IV ${ }^{23,33}$ and should therefore been tested for the more complex flow conditions as presented in this paper.

\section{Steady Results}

\section{IV.A. Convergence Behaviour}

All computations are converged to ensure that $C_{L}$ and $C_{D}$ do not vary more than $\Delta C_{L} \leq \pm 10^{-4}$ and $\Delta C_{D} \leq \pm 10^{-5}$ within the last 500 iterations. A decay of the density residual of about seven orders of magnitude was achieved with the SA and SST turbulence models and three orders of magnitude with the RSM model. For all grids, a $y^{+}<1$ has largely been achieved. A maximum value of $y^{+}=1.6$ was observed on the coarse grid.

\section{IV.B. Grid Convergence Study}

Figures 5 and 6 give an overview on lift, drag and pitching moment coefficients obtained with the different turbulence models for the members of the grid family. As depicted a well-converged solution on the fine grid was not achieved with the RSM model. A similar situation has been observed in DLR's work for DPW-4. 


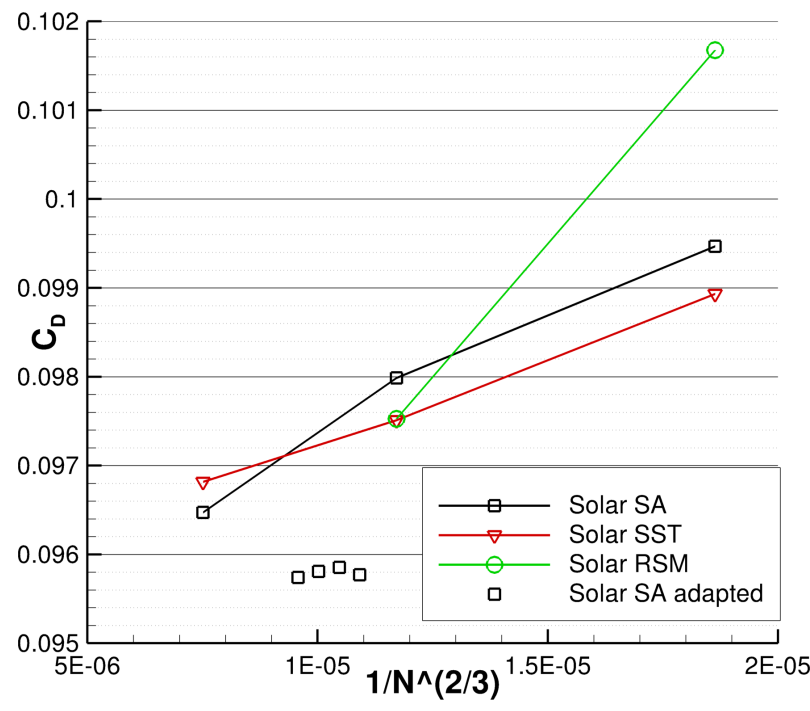

(a) Drag coefficient.

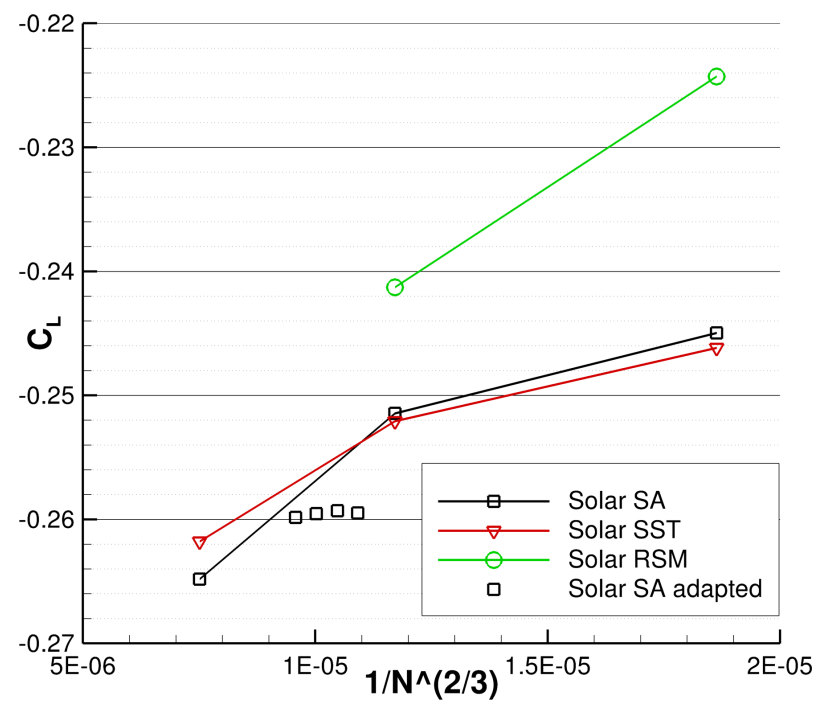

(b) Lift coefficient.

Figure 5. Results of the grid convergence and grid adaptation studies.

The reason for this is not yet fully understood and subject to ongoing work.

Figure 5(a) depicts the influences of mesh resolution and turbulence model on the drag coefficient. For the coarse grid, the RSM model predicts the highest drag, which is approx. $2 \%$ higher than the SA result. The SST model predicts the lowest drag, with minor differences only compared to the SA model (-0.5\%). Regarding the medium grid the results are within a much closer range. The SA model now predicts highest drag, followed by the RSM and SST models. Significant here is the strong decline in drag predicted by the RSM model when compared to the coarse grid results $(-4.1 \%)$. The reason for this consists in a more distinct change of the wing pressure distribution, especially in the vicinity of the spoilers, in case of the RSM model compared to the two other models. A more profound insight into this is given in section IV.E. The RSM model now predicts a nearly identical drag as the SST model. The offset between SA and SST results remains constant, with a drag reduction of $1.5 \%$ compared to the coarse grid. In contrast to the coarser grids, the SST model predicts a slightly higher drag than the SA model on the fine grid. The offset between both, however, has reduced. A look at the curve slope of the SST results reveals a nearly constant gradient, indicating that the solution is within the asymptotic range of convergence. Therefore, an almost second order drag convergence behaviour was reached for the computations using the SST model. Employing a Richardson extrapolation between medium and fine grids results in a drag coefficient of $C_{D, R}=965.9 \mathrm{dc}$, which is less than $1 \%$ below the result of the medium grid.

Figure 5(b) shows the influences of mesh resolution and turbulence model on the lift coefficient. Independent of the mesh resolution, all turbulence models predict a negative lift force for the given onflow conditions. In general, the RSM model predicts higher (more positive) lift forces than the SA and SST models. The latter reveal fairly identical results with a maximum deviation of approx. $1 \%$ observed for the fine grid. In compliance with the observations in the drag behaviour, the SST model predicts a higher lift than the SA model in case of the fine grid only. Furthermore, when comparing figures 5(a) and 5(b) it becomes evident that a more positive lift coefficient does not automatically imply a higher drag coefficient, as shown by the RSM results on the medium grid. The reason for the latter consists in a different prediction of the shock location between the RSM and the SA/SST models. In addition, the SA/SST models show regions of flow separation where the RSM model does not. A closer look into detail is given in section IV.E.

Figure 6 depicts the influences of mesh resolution and turbulence model on the pitching moment coefficient. All turbulence models predict a positive pitching moment (pitch-up tendency). As the RSM model predicts more positive lift coefficients than the eddy-viscosity models, it shows a less distinct pitch-up tendency. As stated earlier, the reason for this mainly consists in different shock positions. The SST model, for instance, always predicts the most upstream shock position on wing and HTP, resulting in a loss of lift force and a higher pitch-up moment. In contrast, the RSM model permanently predicts the most downstream shock position, leading to higher lift forces and a lower pitch-up tendency. 


\section{IV.C. Grid adaptation}

The global lift, drag and pitching moment solutions after each of the four grid adaptation cycles is depicted in figures 5 and 6 . It becomes evident that the most significant changes compared to the medium-sized SA reference solution take place after the first grid adaptation cycle. The nearly $11 \%$ increase in total grid points after the first cycle leads to a decline of $2.3 \%$ in drag coefficient and $3.2 \%$ in lift coefficient, whereas the pitching moment augments by $3 \%$. The variations in the solutions after the remaining three adaptation cycles remain negligible, an almost grid-independent behaviour is observed. As a consequence the grid adaptation cycles employing the vortex detection sensor did not contribute to a further enhancement of the solution quality.

Nevertheless, the procedure applied in the first adaptation cycle reveals an improved solution quality as the results do approach the results of the fine grid. The grid adaptation procedure reveals its potential in adding $11 \%$ new points only to the medium reference grid and, simultaneously, reducing the differ-

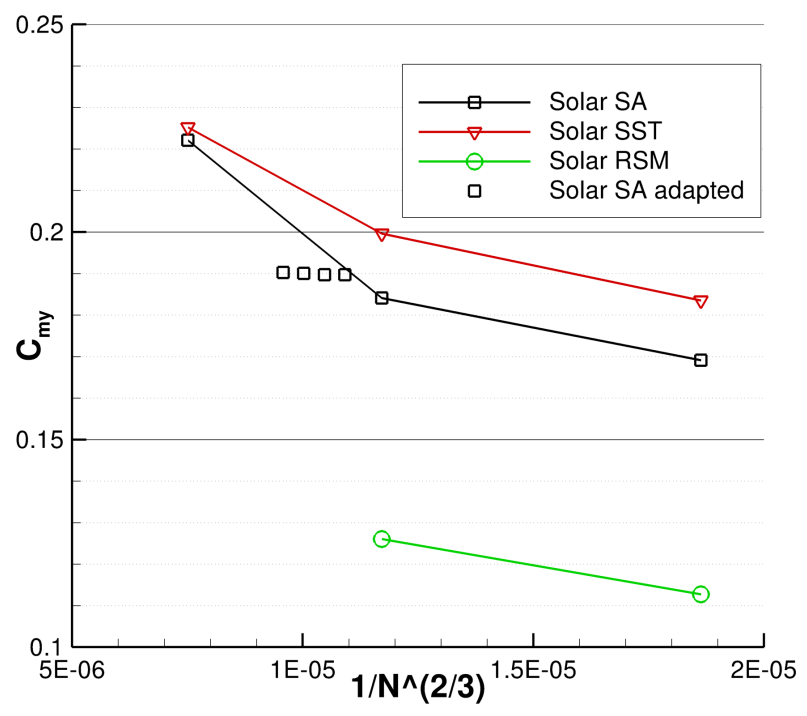

Figure 6. Results of the pitching moment coefficient during the grid convergence and grid adaptation studies. ences compared to the fine grid solution from $5 \%$ to $2 \%$ for $C_{L}$, from $2 \%$ to $0.7 \%$ for $C_{D}$ and from $17 \%$ to $14 \%$ for $C_{m y}$.

\section{IV.D. Aerodynamic Load Distributions}

The computations have been evaluated with focus on the aerodynamic load distribution of wing and HTP. These results are depicted in figure 7 for both configurations. Regarding the clean wing in figure 7 (a) the influence of the turbulence model with respect to the spanwise load distribution is negligible. At first glance, the same holds for the spoiler configuration as depicted in figure 7(b). A closer look in the vicinity of the spoilers, however, reveals a slightly different load distribution. As will be shown in a later section, this is mainly attributed to different predictions of the shock positions as well as different pressure levels, especially behind the spoilers.

Figure 7(c) shows the spanwise HTP load distribution of the clean configuration, revealing a significant influence of the turbulence model. The SA and SST models show a qualitatively similar load distribution, however, with absolute differences near the HTP-fuselage intersection as well as at mid-span. The reason for this consists in the different sensitivity in predicting the shock-induced boundary layer separation on the HTP. Furthermore, the mesh resolution in the concave corner between fuselage and HTP might not be sufficient to accurately capture the relevant flow features. A further examination will take place. In general, both models predict a net downward force acting on the HTP whereas the RSM model predicts a net upward force as listed in table 5. Furthermore, in case of the RSM model the HTP is producing lift over its entire span. The reason for the distinct peak at the inboard section of the HTP is attributed to the fact that the RSM model, in contrast to the eddy-viscosity models, shows purely attached flow along the intersection of fuselage and HTP.

A comparison of the HTP load distributions in figures 7(c) and 7(d) reveals only minor differences between the two configurations. The offset between the turbulence models nearly remains constant. Nonetheless, all curves are shifted towards more positive lift forces which can be traced back to different local HTP onflow conditions due to interference effects with the wake vortices of the inboard spoiler.

\section{IV.D.1. Influence of the Mesh Resolution}

Based on the solution of the different meshes used in the grid convergence study the influence of the mesh resolution on the load distributions of the spoiler configuration are examined. As shown in table 5 and figure 8 the HTP and wing load distributions are shifted towards smaller $z$-forces for increasing mesh resolution. As for the wing the results of the SA and SST models remain close to each other on all meshes. 


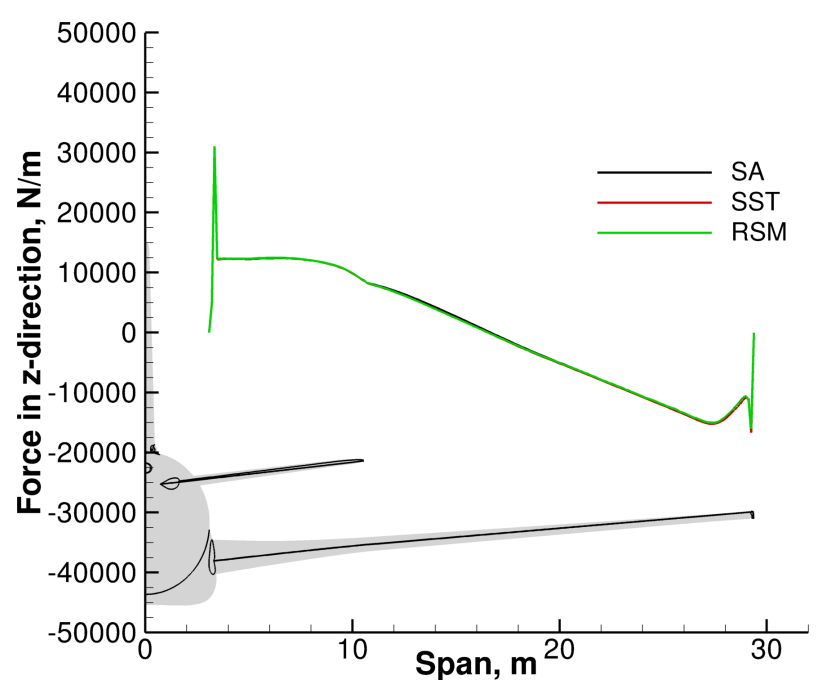

(a) Wing load distribution of the clean configuration.

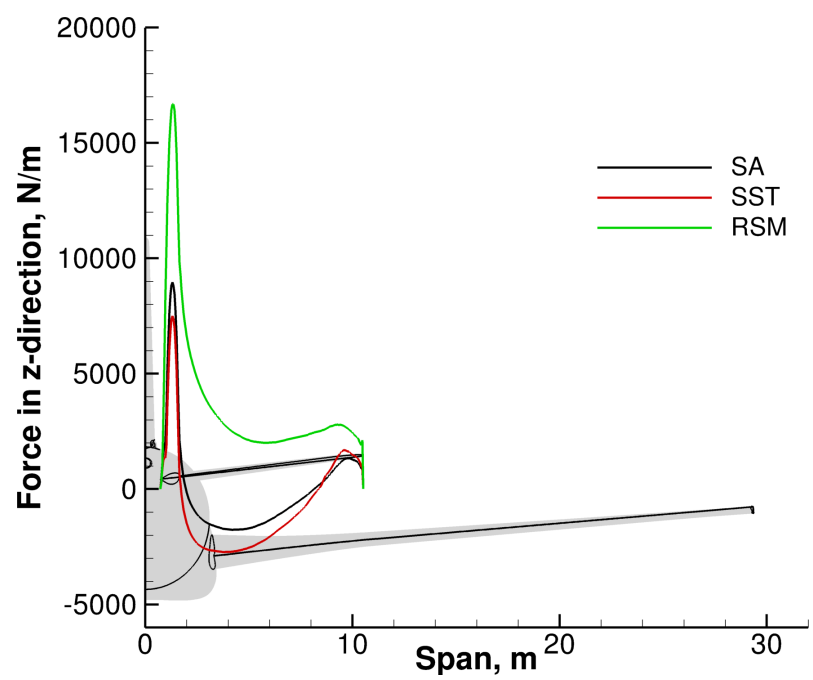

(c) HTP load distribution of the clean configuration.

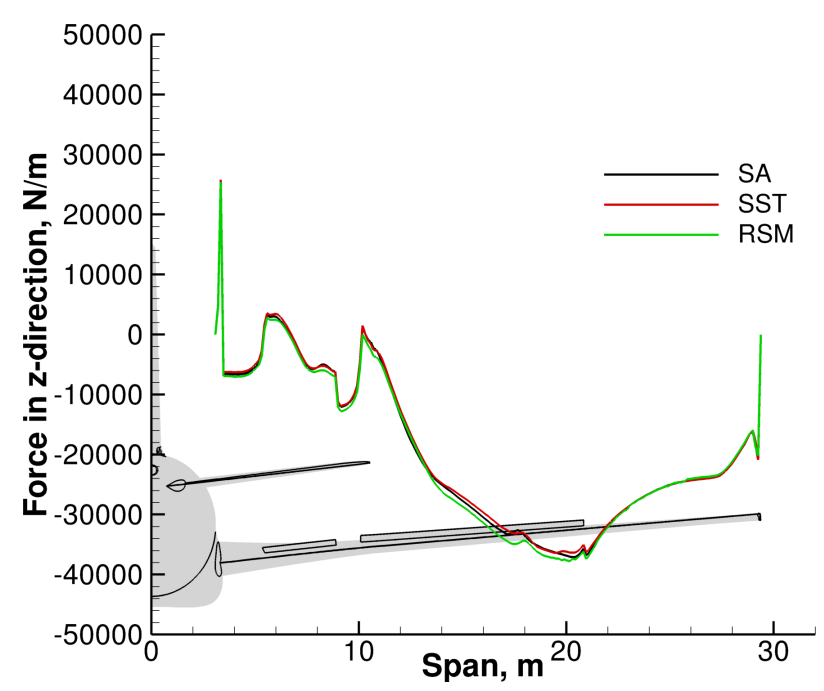

(b) Wing load distribution of the spoiler configuration.

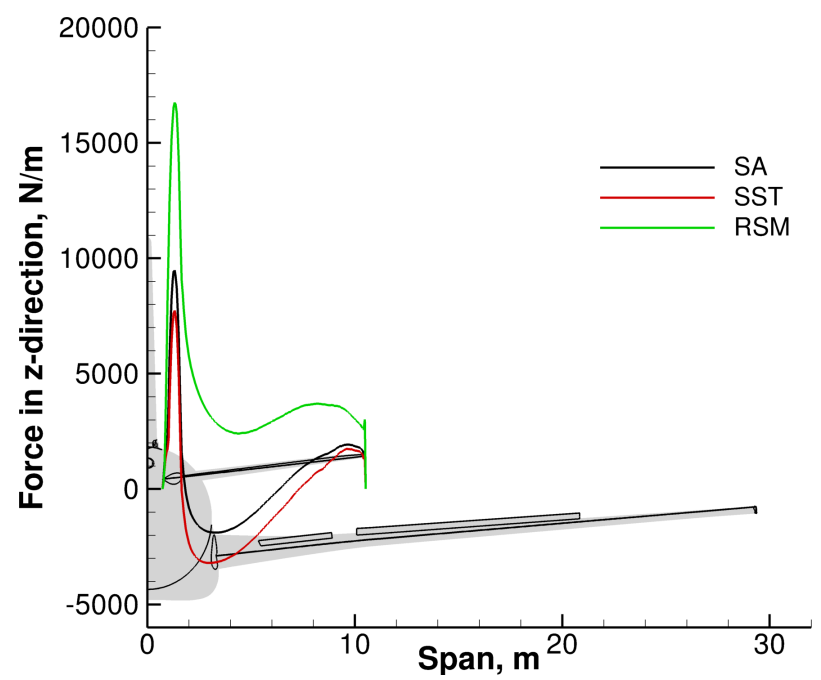

(d) HTP load distribution of the spoiler configuration.

Figure 7. Spanwise load distributions on wing (top) and HTP (bottom) based on the medium-sized grids.

Table 5. Comparison of the integrated forces in $z$-direction acting on wing and HTP. For the spoiler configuration the data is provided for the coarse $(\mathrm{c})$, the medium $(\mathrm{m})$ and the fine grid $(\mathrm{f})$.

\begin{tabular}{lcccc}
\hline \hline Configuration (mesh size) & & SA [kN] & SST [kN] & RSM [kN] \\
\hline \multirow{2}{*}{ Clean (m) } & Wing & 7.2 & 4.3 & 6.3 \\
& HTP & -0.5 & -6.8 & 36.3 \\
\hline \multirow{2}{*}{ Spoiler (c) } & Wing & -504.7 & -500.8 & -475.1 \\
& HTP & 12.1 & 3.6 & 42.1 \\
\hline \multirow{2}{*}{ Spoiler (m) } & Wing & -519.4 & -512.9 & -531.4 \\
& HTP & 4.7 & -4.6 & 40.1 \\
\hline \multirow{2}{*}{ Spoiler (f) } & Wing & -534.7 & -522.9 & - \\
& HTP & -15.9 & -18.7 & - \\
\hline \hline
\end{tabular}




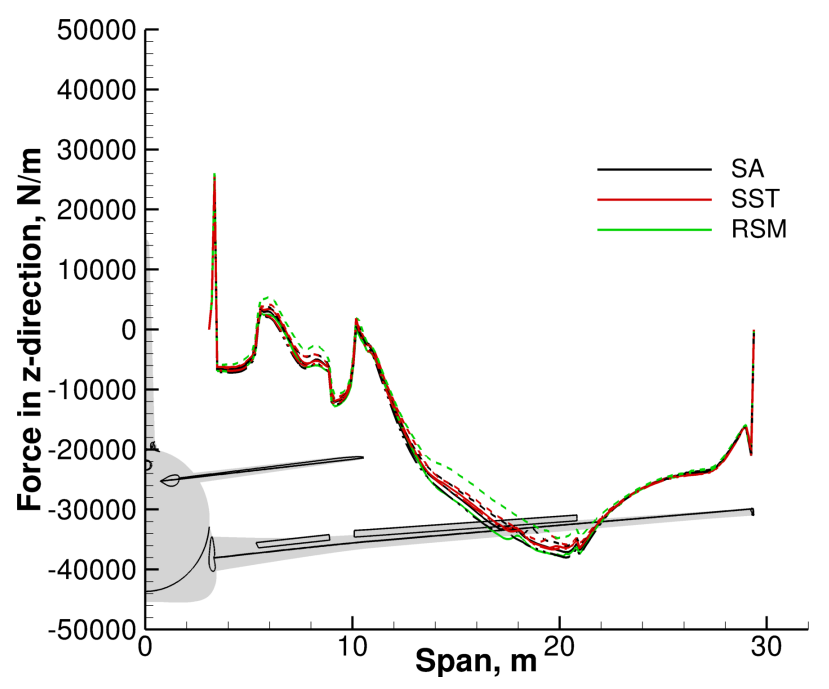

(a) Wing load distribution.

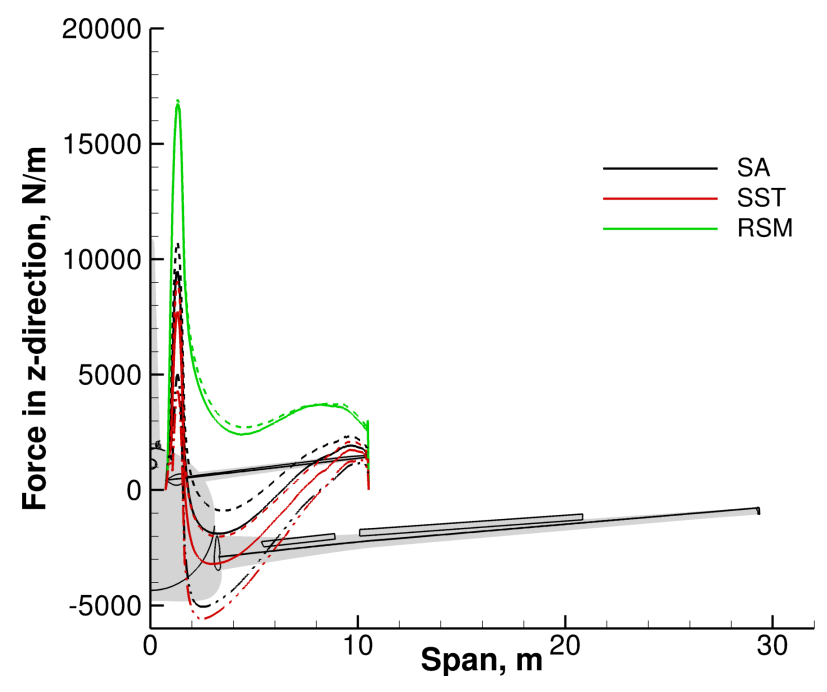

(b) HTP load distribution.

Figure 8. Influence of the mesh resolution to the spanwise load distributions of wing and HTP. Illustrated are the results of the coarse grid $(---)$, of the medium grid $(-)$ and of the fine grid $(----)$.

The most significant changes occur for the RSM model from coarse to medium grid, especially in the vicinity of the spoilers. The SA result on the fine grid is almost identical to the RSM result on the medium grid.

The HTP load distribution massively depends on the mesh resolution for the SA and SST models. In analogy to the behaviour observed at the wing the load distribution is shifted towards more negative forces when increasing the mesh resolution. As given in table 5 the SST model always indicates the highest downward forces, whereas the RSM model always predicts upward forces. The results of the SA model lie in between. The reason for the latter consists in different shock positions. As stated earlier the SST model always predicts the shock at the most upstream position, whereas the shock is located at the most downstream position when using the RSM model.

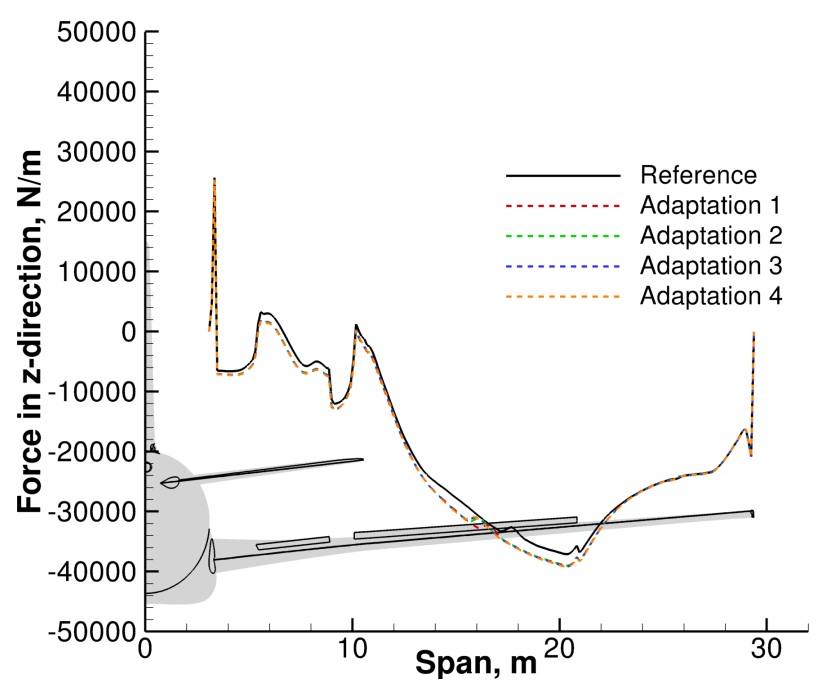

(a) Wing load distribution.

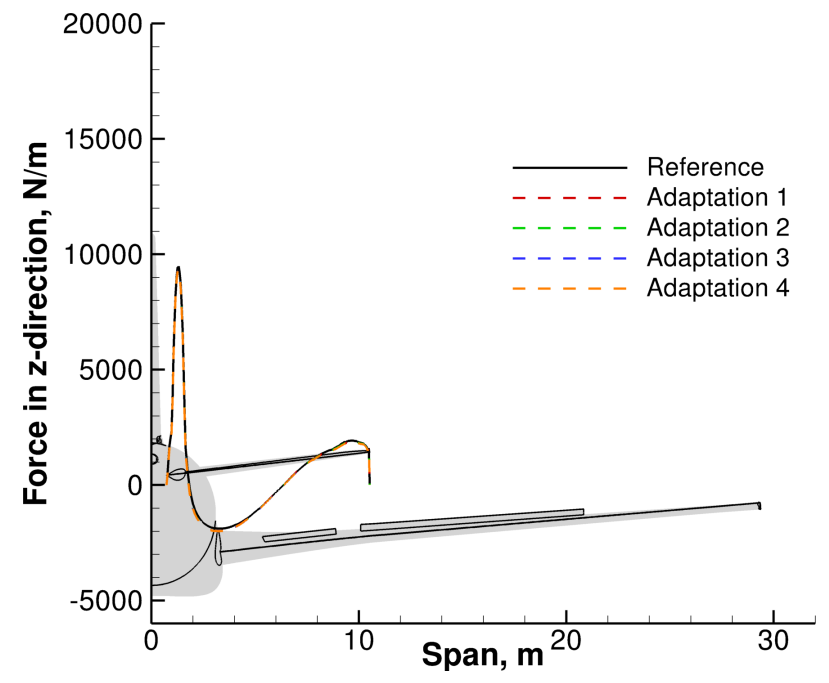

(b) HTP load distribution.

Figure 9. Influence of the grid adaptation procedure to the spanwise load distributions of wing and HTP. Illustrated are the results of the medium reference grid $(-)$ and of the different adaptation cycles (- - -). 


\section{IV.D.2. Influence of the Grid Adaptation}

Figure 9 shows the influence of the mesh adaptation on the spanwise load distributions of wing and HTP. As depicted in figure 9(a) the wing load distribution shows significant changes after the first adaptation cycle as the curve is shifted towards more negative forces, which is in compliance with the observations of the grid convergence study. In particular this can be recognized near the spoilers due to a refined mesh resolution in the shock region. The changes for spanwise positions beyond the outboard spoiler are negligible. The same holds for the variations from the first to the fourth adaptation cycle.

The HTP load distribution shows minor changes only. In compliance with the observations on the wing the HTP load distribution is slightly shifted towards more negative forces after the first adaptation cycle. The distribution is unaffected from the remaining adaptation cycles.

\section{IV.E. Detailed Flow Analysis}

The analysis in this section focusses on the examination of the sectional pressure distributions on wing and HTP. In addition, the skin friction lines are evaluated to visualize the three-dimensional turbulence model influence on the near-wall flow topology.

\section{IV.E.1. Sectional Wing Pressure Distributions}

For the visualization of the sectional $C_{p}$-distributions four exemplary cuts were generated based on the medium grid solutions of the spoiler configuration. The wing cuts are located at spanwise positions of $y=5 \mathrm{~m}, 7.5 \mathrm{~m}, 15 \mathrm{~m}$ and $28 \mathrm{~m}$. The two inner positions include the spoilers as shown in figure 10 .

The exemplary results of the wing pressure distribution is given in figure 11. For the inner cut shown in figure 11(a) the pressure on the lower wing side is equally captured by all turbulence models. In general the same holds for the upper wing side. Minor differences in the shock position and in the following pressure rise can be recognized.

The pressure distribution at the location of the inboard spoiler as depicted in figure 11(b) is identical on the lower wing side. Regarding the upper side the position and strength of the shock is comparable, however with differences occurring in the pressure rise behind the shock. The SST model predicts a lower pressure rise in front of and on the spoiler than the SA and RSM models. In addition, slight differences can be found behind the spoiler, just in front of the trailing edge of the wing where the SST and RSM results are in good agreement. The SA model results are marginally shifted towards lower pressure levels.

The situation on the lower wing surface at the outboard spoiler is comparable to the inner sections as seen in figure 11(c). The behaviour on the upper side is similar to the behaviour recognized on the upper side of the inboard spoiler, at least for the part in front of the spoiler. The results behind the spoiler increasingly distinguish, with the SST model again predicting the lowest pressures. The SA results match the SST results very well, the RSM model predicts a reduced low-pressure region compared to the eddy-viscosity models. These findings are reflected in the overall drag coefficient as mentioned earlier in section IV.B.

The aforementioned also becomes evident for the most outboard cut as shown in figure 11(d). Apart from the stronger suction peak in case of the SA and RSM models, significantly different pressure levels can be recognized within the last $25 \%$ of the chord length. Again, the SST model predicts lower pressure levels than the SA and RSM models. 


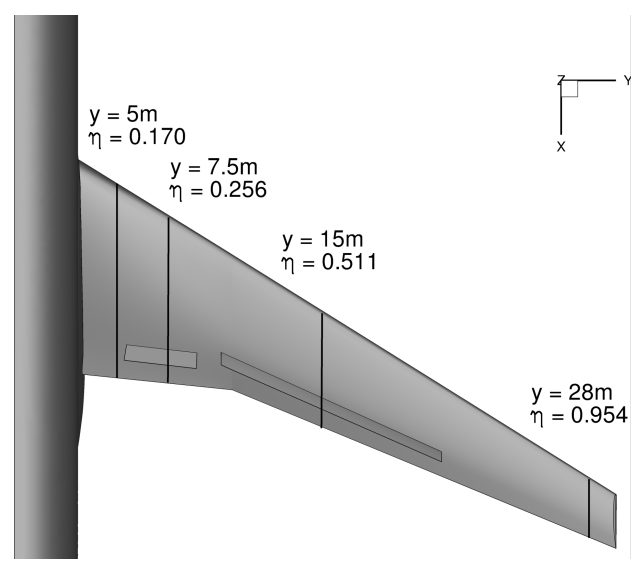

(a) Wing.

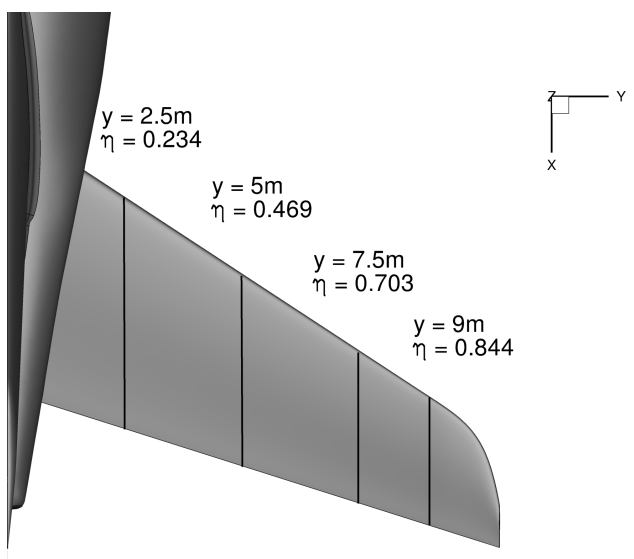

(b) HTP.

Figure 10. Location of the four spanwise cuts on wing and HTP to derive the sectional $C_{p}$-distributions.

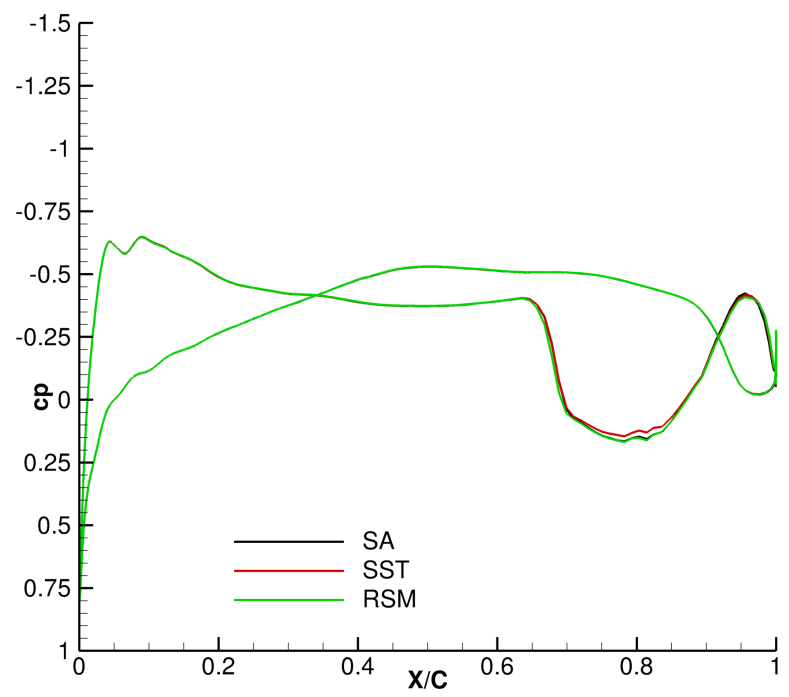

(a) Wing cut at $y=5 \mathrm{~m}$.

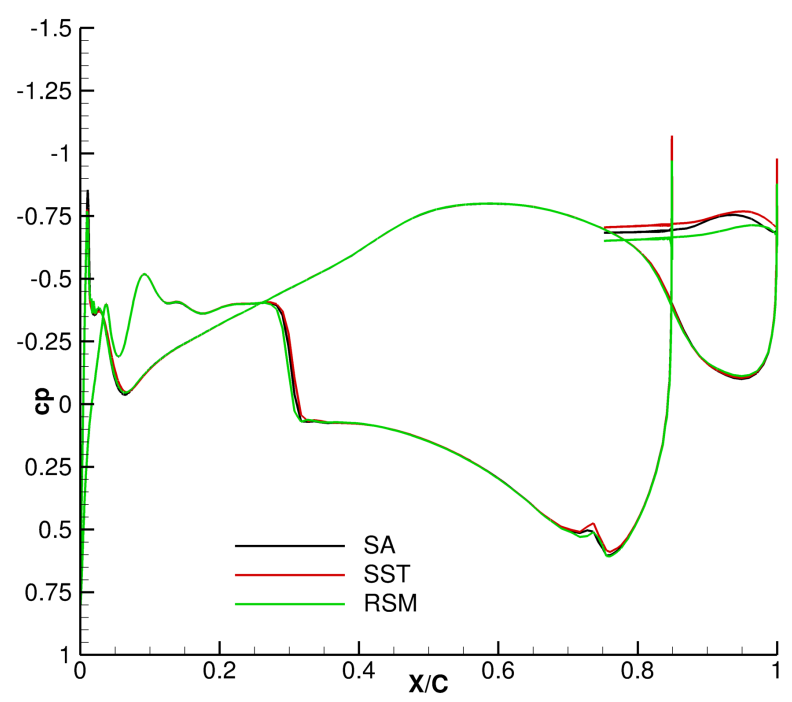

(c) Wing cut at $y=15 \mathrm{~m}$.

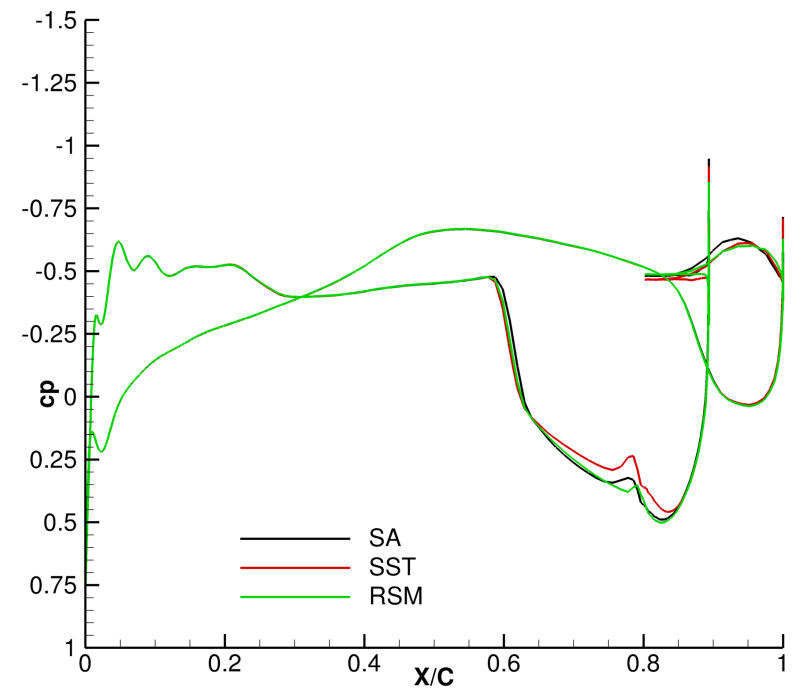

(b) Wing cut at $y=7.5 \mathrm{~m}$.

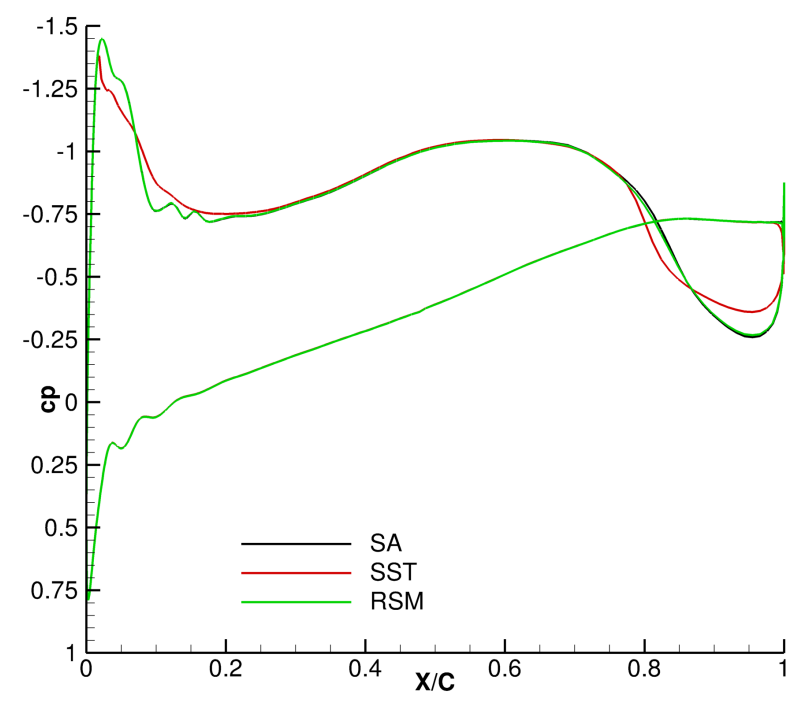

(d) Wing cut at $y=28 \mathrm{~m}$.

Figure 11. Wing $C_{p}$-distributions at four spanwise cuts. 


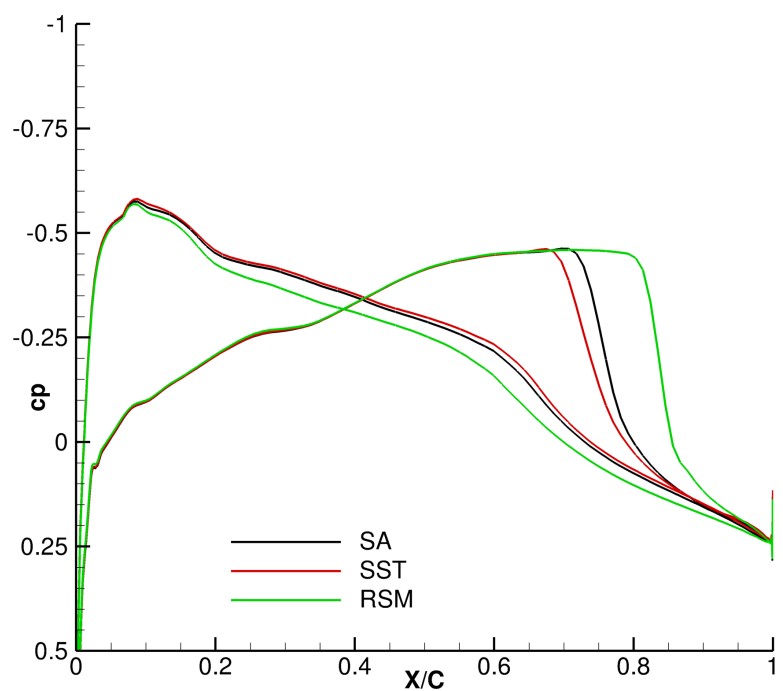

(a) HTP cut at $y=2.5 \mathrm{~m}$.

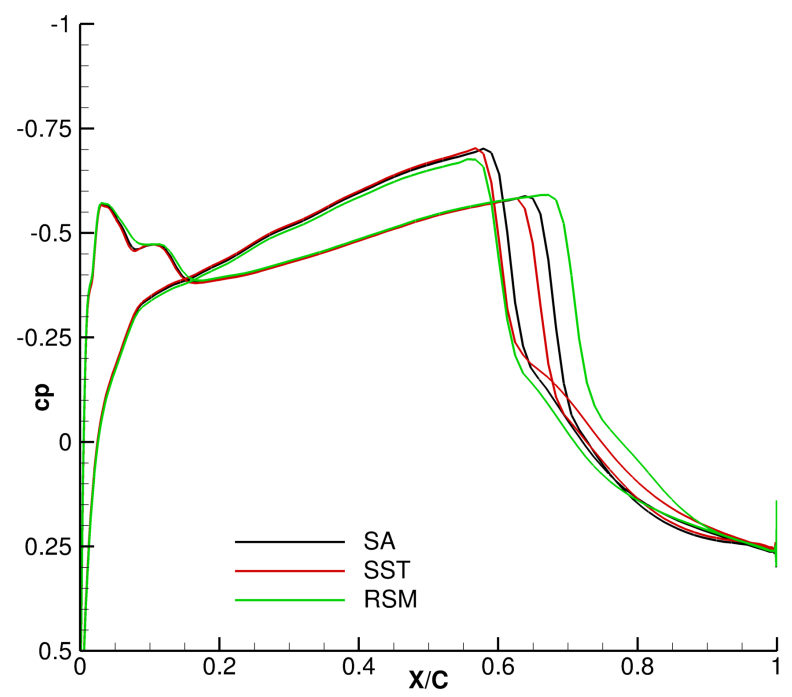

(c) HTP cut at $y=7.5 \mathrm{~m}$.

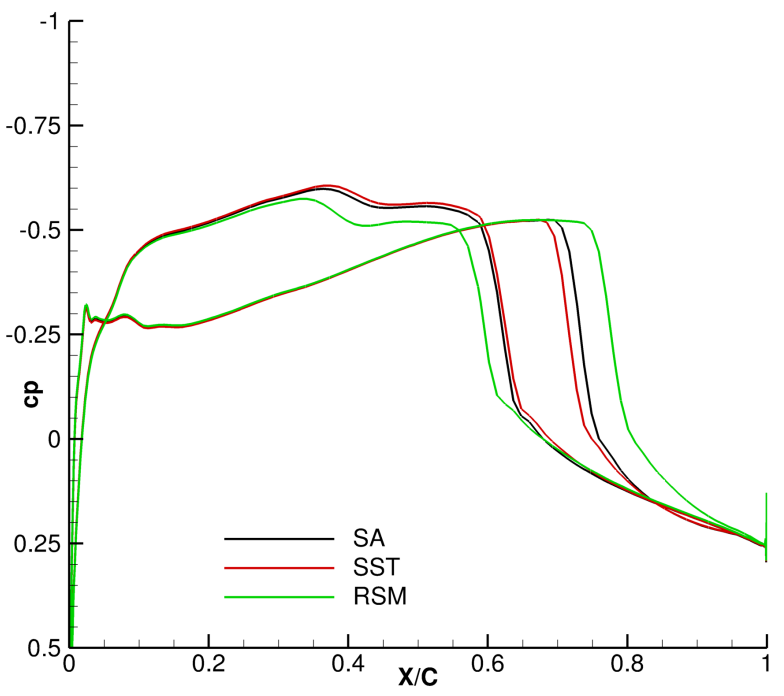

(b) HTP cut at $y=5 \mathrm{~m}$.

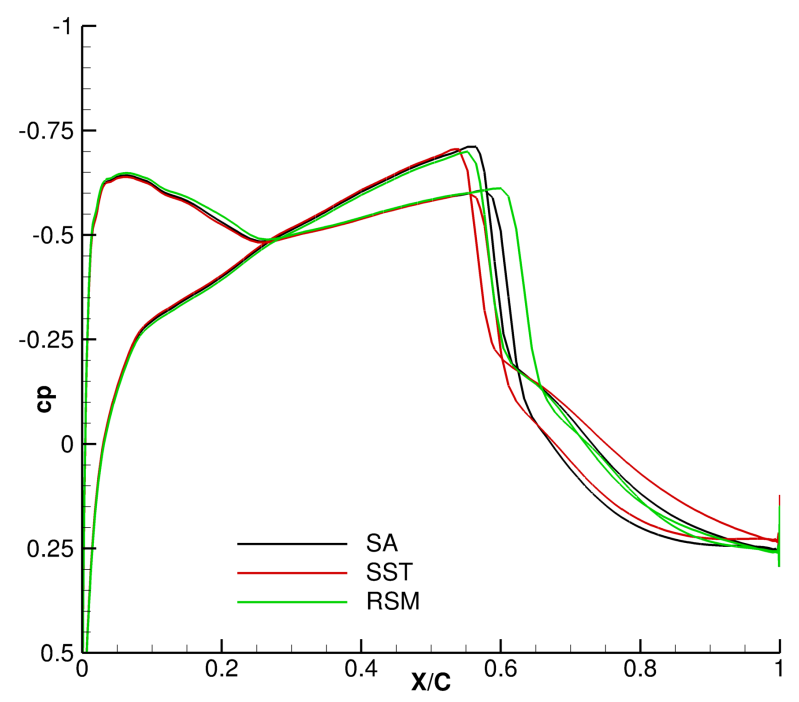

(d) HTP cut at $y=9 \mathrm{~m}$.

Figure 12. HTP $C_{p}$-distributions at four spanwise cuts.

\section{IV.E.2. Sectional HTP Pressure Distributions}

Figure 10(b) depicts the positions of the HTP cuts. For the inboard cut shown in figure 12(a) the pressure distribution on the lower side is nearly identical between SA and SST models. The RSM models reveals a qualitatively equal behaviour, however, beginning from the suction peak onwards, the RSM solution is shifted towards more positive pressure levels. On the upper side all turbulence models predict the same pressure distribution up to the shock location. Here, the SST models predicts the shock position at $x / c=70 \%$, the SA model a bit farther downstream at $x / c=72 \%$. The RSM model, in contrast, shows a much larger plateau of nearly constant pressure before the shock occurs at $x / c=82 \%$. These significant discrepancies in the prediction of the shock position are one reason why the spanwise load distribution of the RSM model massively differs from the one of the eddy-viscosity models as shown above in figures 7 (c) and $7(\mathrm{~d})$.

Regarding the cut at $y=5 \mathrm{~m}$ in figure $12(\mathrm{~b})$ shocks on both the upper and lower side of the HTP can be recognized. On the lower side, the RSM model is shifted again towards more positive pressure levels, yielding a more forward shock position. On the upper side, the sequence of the models in predicting the shock position remains the same as on the inboard cut. 
Nevertheless, all turbulence models predict an upstream movement of the shock compared to the inboard cut. In addition, the gap between the RSM model and the SA/SST models becomes smaller.

The pressure distribution at $y=7.5 \mathrm{~m}$ in figure 12 (c) reveals a stronger shock on the lower HTP side. Furthermore, the nearly constant pressure level seen on the lower side of the cut at $y=5 \mathrm{~m}$ has vanished. Instead, an extensive low-pressure area has developed, yielding a strong shock at $x / c=72 \%$. The location of the shock position of SST and RSM models is in good agreement. However, it can be recognized that the pressure increase after the shock is more distinctly developed in case of the RSM model. The situation on the upper side is similar to the aforementioned findings. The shock position, however, has moved in upstream direction again.

The pressure distribution of the outboard cut at $y=9 \mathrm{~m}$ in figure $12(\mathrm{~d})$ is dominated by two strong shocks at the lower and upper side of the HTP, occurring at $x / c \approx 60 \%$ each. No significant changes to the already mentioned facts occur.

\section{IV.E.3. Skin friction Lines}

Skin friction lines are used to visualize regions of detached flow on the wing and on the empennage. Figures 13 and 14 show the turbulence model influence on the skin friction lines for the medium-sized spoiler grid.

As indicated by the curvature of the skin friction lines on the empennage three main regions of flow detachment can be recognized. The flow detachment on the tip of the VTP is equally predicted in location and extension by the SA and SST models. The RSM models shows a reduced size of this area. The same holds for the shock-induced flow detachment in the tip region of the HTP. The main difference, though, consists in the flow detachment area near the inner HTP trailing edge region and the HTP-fuselage junction. The eddy-viscosity models reveal a distinct flow separation on the rear fuselage as well as on the HTP. For the SST model, the size of the latter is a bit larger than the one computed with the SA model. The different sizes of these regions are reflected in the spanwise load distribution of the HTP as shown in figures 7 (c) and 7(d) where the SA model is producing higher peak forces in that region. The RSM model, in contrast, seems to show no flow separation in this region, neither on the fuselage nor on the HTP, which leads to a tremendously different spanwise load distribution as discussed earlier. In fact, a magnified view into this region of interest also revealed a tiny area of flow separation, which, however, is restricted to the last $10 \%$ of the HTP chord length and showing a very small extension in lateral direction only.

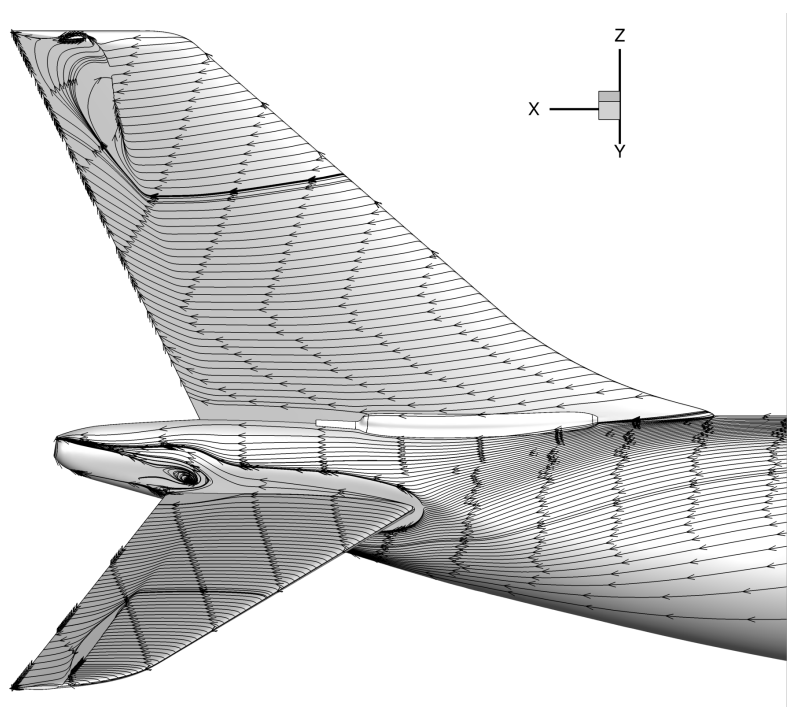

(a) Tail section (SA).

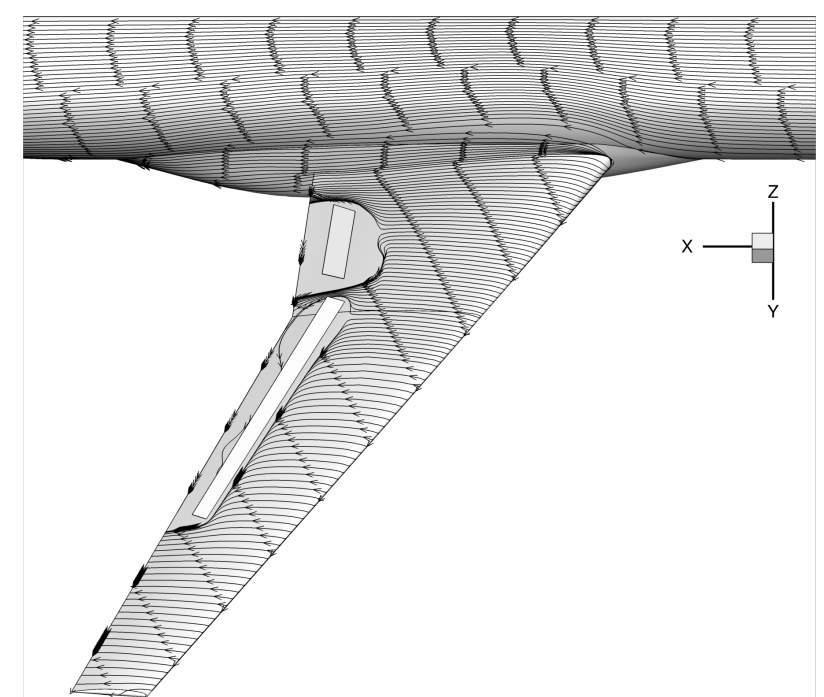

(b) Wing section (SA).

Figure 13. Visualization of skin friction lines on the empennage and the wing for the SA turbulence model. 


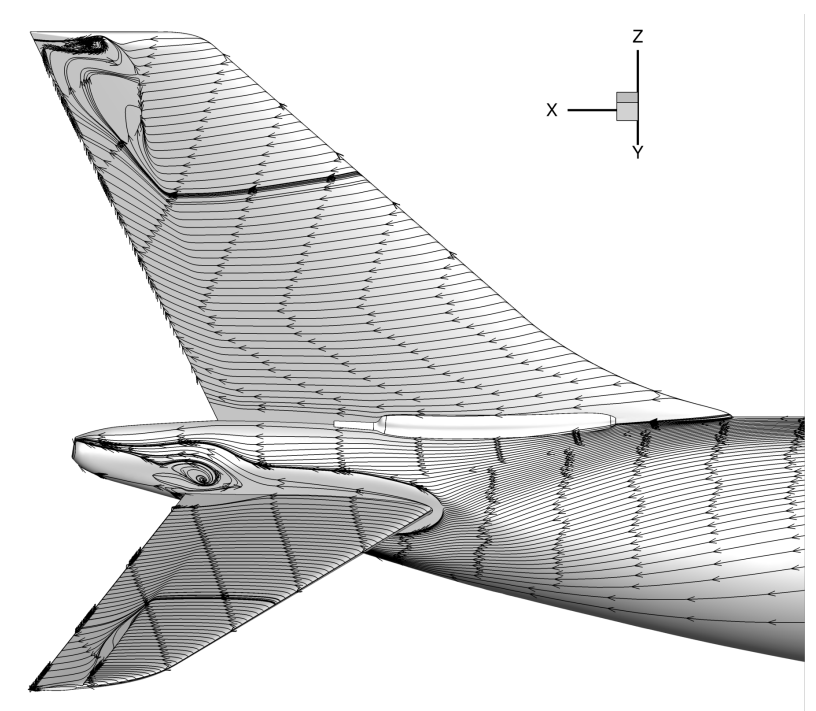

(a) Tail section (SST).

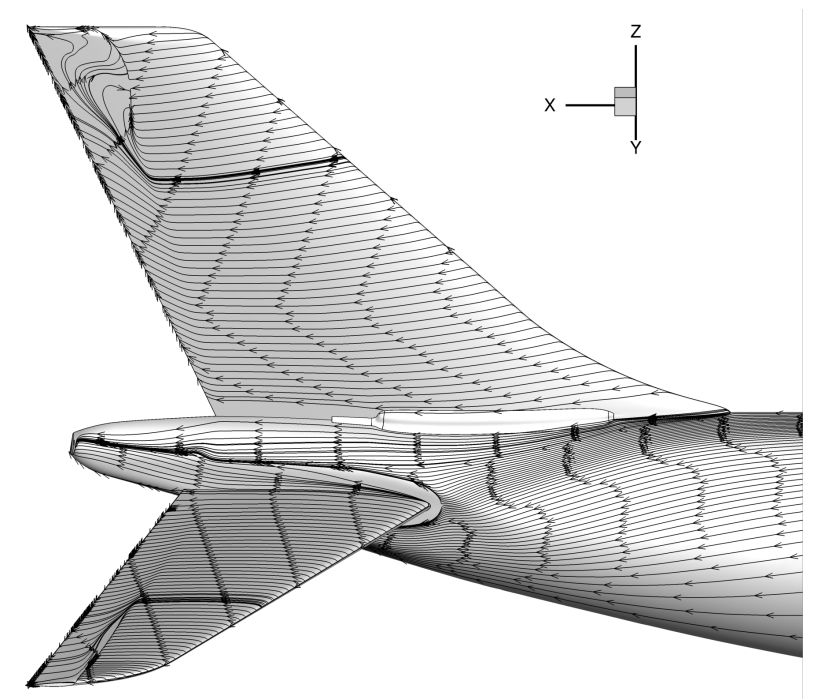

(c) Tail section (RSM).

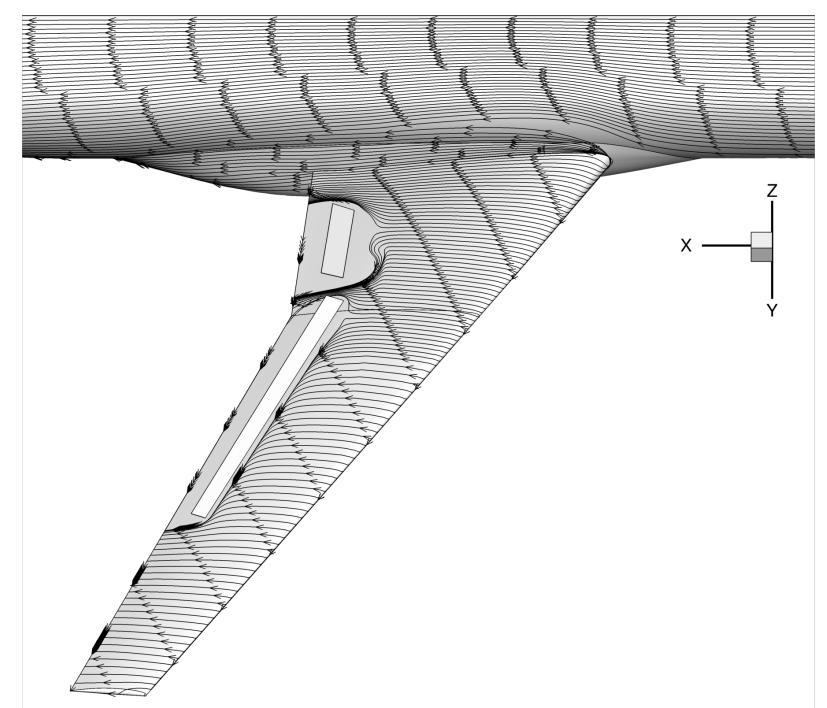

(b) Wing section (SST).

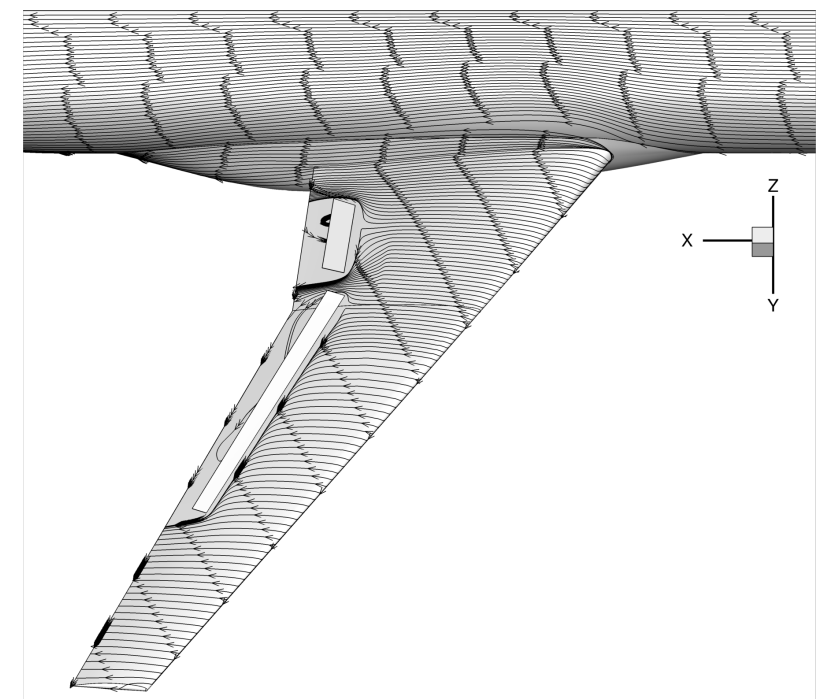

(d) Wing section (RSM).

Figure 14. Visualization of skin friction lines on the empennage and the wing for the SST and RSM turbulence models.

\section{Unsteady results}

Time-dependent (uRANS) computations were exemplarily performed to capture the highly vortical, unsteady flow field behind the wing and to assess the differences in the global force and moment coefficients compared to the steady RANS approach. The unsteady computations were carried out for both the clean wing reference configuration and the spoiler configuration. For each configuration, three unsteady computations with different time steps sizes were computed. All computations were restart computations based on a well-converged SST solution on a respective medium-sized grid.

\section{V.A. Determination of Time Step Size}

To derive a time step size $\Delta t$ for the unsteady computation a characteristic time $t^{*}$ tailored to the particular needs in this study was determined as given by Eq. (1), with $v_{\infty}$ being the onflow velocity. As a main focus within this work consists in evaluating interference effects of the spoiler wake with the HTP, the characteristic length $l_{\text {ref }}$ in Eq. (1) was chosen such to represent the distance between wing leading edge to HTP leading edge. 
This distance was determined at a spanwise position of $y=7.5 \mathrm{~m}$ (mid of inboard spoiler), yielding a length of $l_{\text {ref }}=36 \mathrm{~m}$. Using this data the characteristic time was determined as $t^{*}=126.44 \mathrm{~ms}$. Assuming that $v_{x} \gg v_{y}, v_{z}$ the characteristic time $t^{*}$ represents the approximate time for a fluid particle to travel from the wing leading edge downstream to the leading edge of the HTP. This "motion" was then discretized with 100 time steps, yielding the initial time step size $\Delta t_{i}=1.264 \mathrm{~ms}$.

$$
t^{*}=\frac{l_{r e f}}{v_{\infty}}
$$

\section{V.B. Variation of Time Step Size}

In order to examine the sensitivity of the simulation results with respect to the magnitude of the time step a variation of the time step size has been conducted. Based on the initial time step size $\Delta t_{i}$ a ten times smaller time step size $\Delta t_{s}=0.1264 \mathrm{~ms}$ and a ten times larger time step size $\Delta t_{l}=12.64 \mathrm{~ms}$ have been derived and used during computation. For each time step size 700 unsteady time steps were computed.

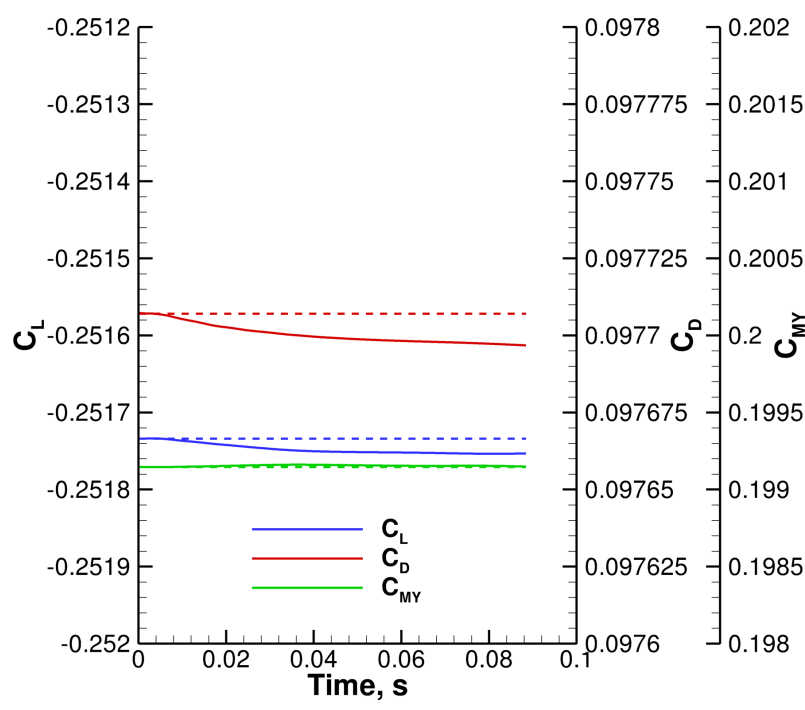

(a) $\Delta t_{s}=0.1264 \mathrm{~ms}$.

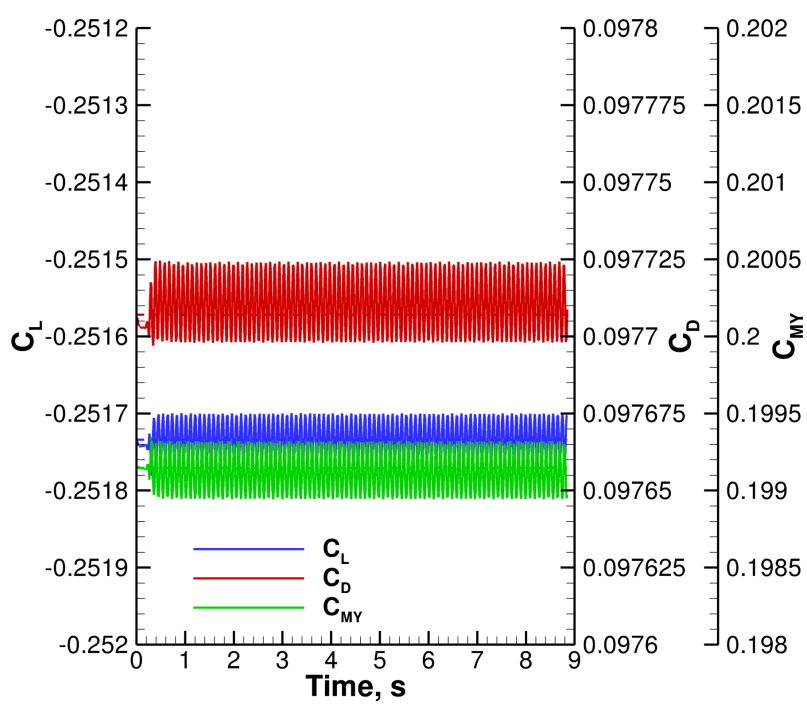

(c) $\Delta t_{l}=12.64 \mathrm{~ms}$.

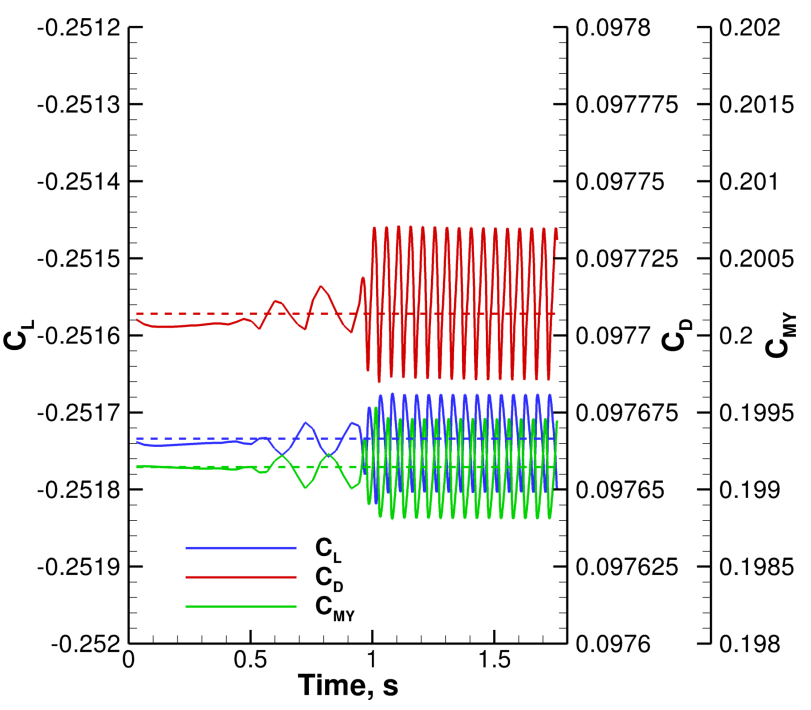

(b) $\Delta t_{i}=1.264 \mathrm{~ms}$.

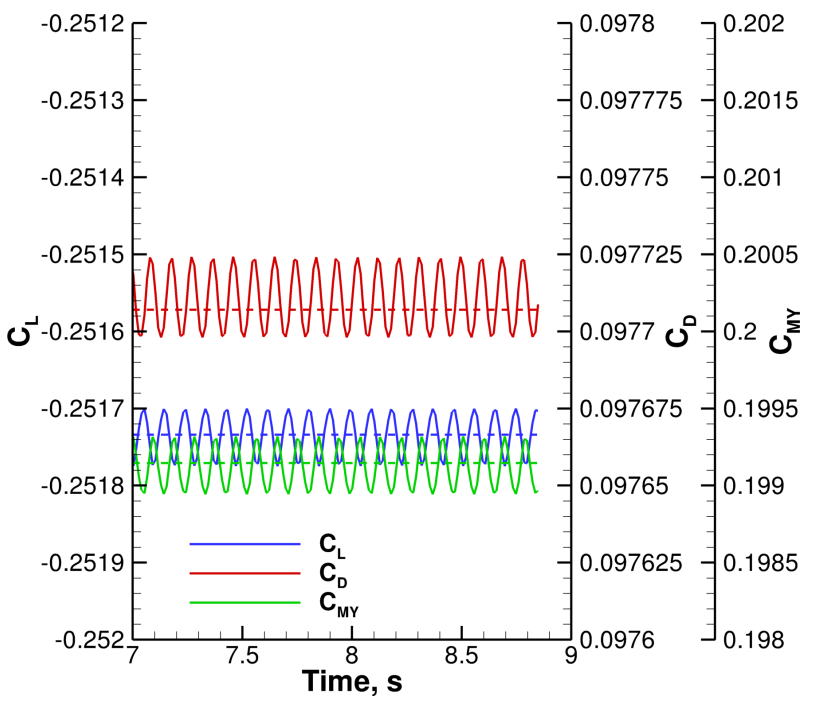

(d) $\Delta t_{l}=12.64 \mathrm{~ms}$, last $2.8 \mathrm{~s}$.

Figure 15. Convergence behaviour of uRANS (-) computations compared to steady RANS (- - -) results. 
The computations with the initial time step size $\Delta t_{i}$, however, were started with 30 larger time steps until $t=0.945 \mathrm{~s}$ before switching to $\Delta t_{i}=1.264 \mathrm{~ms}$.

\section{V.C. Influence on Convergence Behaviour}

Figures 15(a)-15(d) show the unsteady convergence behaviour of lift, drag and pitching moment coefficient compared to the steady solution. Figure 15(d) represents a zoomed view into the last $2.8 \mathrm{~s}$ for the large time step $t_{l}=12.64 \mathrm{~ms}$. Shown are the unsteady results for the spoiler configuration only as the results of the clean configuration show a similar behaviour.

As shown, the differences between the mean values of the uRANS results and the steady results are negligible. This behaviour is in agreement with observations for a similar configuration as published in Ref. 34. For the smallest time step size no oscillatory behaviour is observed within the computed time interval (figure 15(a)). The two larger time step sizes lead to a stable oscillation with constant amplitude. The oscillation frequencies amount to $f_{i} \approx 20 \mathrm{~Hz}$ at $\Delta t_{i}=1.264 \mathrm{~ms}$ and $f_{l} \approx 11 \mathrm{~Hz}$ at $\Delta t_{l}=12.64 \mathrm{~ms}$. Table 6 summarizes the uRANS results in comparison with the steady solutions.

Table 6. Comparison of RANS and uRANS results for the spoiler configuration.

\begin{tabular}{lccc}
\hline \hline Methodology & $C_{L}[\mathrm{lc}]$ & $C_{D}[\mathrm{dc}]$ & $C_{m y}$ \\
\hline RANS & -251.73 & 977.07 & $199.15 \cdot 10^{-3}$ \\
uRANS $\Delta t_{s}=0.1264 \mathrm{~ms}$ & -251.75 & 976.97 & $199.15 \cdot 10^{-3}$ \\
uRANS $\Delta t_{i}=1.264 \mathrm{~ms}$ & $-251.74 \pm 0.06$ & $977.09 \pm 0.26$ & $(199.12 \pm 0.34) \cdot 10^{-3}$ \\
uRANS $\Delta t_{l}=12.64 \mathrm{~ms}$ & $-251.74 \pm 0.04$ & $977.10 \pm 0.14$ & $(199.12 \pm 0.19) \cdot 10^{-3}$ \\
\hline \hline
\end{tabular}

\section{Conclusion and Outlook}

The present work summarizes the results of a numerical feasibility study of a generic transport aircraft configuration with deployed spoilers, carried out at DLR. As an increasing effort has been undertaken to gradually introduce high-fidelity CFD methods for aerodynamic loads prediction, the main focus in this study consisted in assessing the capabilities of prevailing RANS methods for the complex flow cases encountered at the borders of the flight envelope.

In a first attempt, steady RANS methods using a one- and two-equation eddy-viscosity turbulence model as well as a Reynolds stress model were applied to examine the suitability of these methods in off-design flow conditions. Despite the challenging flow conditions, a good convergence behaviour could be observed for nearly all cases throughout this study, which is to a great extent attributed to the highly self-similar grid family generated with SOLAR. Thus, in spite of the massive occurrence of shocks, partially shockinduced flow separation and the highly vortical flow generated by the spoilers, the grid convergence study revealed very promising results. On the other hand, a tremendous influence of the turbulence model could be observed. For instance, the RSM model predicted the shocks on the most downstream positions, leading to completely different load distributions in quantitative terms. In addition to the grid convergence study, the investigation of the influence of a grid adaptation on the load distributions revealed an improved solution quality, at least after the first adaptation cycle.

Finally, time-accurate RANS methods were applied to assess the errors made in applying steady RANS methods for the highly vortical flow field. Based on the investigated onflow conditions, it could be proven that the mean results of the uRANS approach comply with the results of the steady RANS approach. Therefore, when the aerodynamic performance of the aircraft or the mean load distribution are of main interest the steady approach still is applicable.

Based on the results presented within this paper, the study into the suitability of RANS methods will proceed at DLR. As a first step, the detailed flow field analysis will be continued in order to better understand the discrepancies between the turbulence models. Afterwards, the computations will be repeated for the aircraft being in trimmed flight conditions. 


\section{References}

${ }^{1}$ Becker, K. and Vassberg, J., "Numerical Aerodynamics in Transport Aircraft Design," Notes on Numerical Fluid Mechanics and Multidisciplinary Design, edited by E.-H. Hirschel and E. Krause, Vol. 100, Springer, 2009, pp. 209-220.

${ }^{2}$ Rossow, C.-C. and Cambier, L., "European Numerical Aerodynamics Simulation Systems," Notes on Numerical Fluid Mechanics and Multidisciplinary Design, edited by E.-H. Hirschel and E. Krause, Vol. 100, Springer, 2009, pp. 189-208.

${ }^{3}$ Vassberg, J., Tinoco, E., Mani, M., Brodersen, O., Eisfeld, B., Wahls, R., Morrison, J., Zickuhr, T., Laflin, K., and Mavriplis, D., "Abridged Summary of the Third AIAA Computational Fluid Dynamics Drag Prediction Workshop," AIAA Journal of Aircraft, Vol. 45, No. 3, pp. 781-798, 2008.

${ }^{4}$ Han, Z., Görtz, S., and Zimmermann, R., "On Improving Efficiency and Accuracy of Variable-Fidelity Surrogate Modeling in Aero-data for Loads Context," Paper, European Air and Space Conference (CEAS), October 2009.

${ }^{5}$ Mack, M. D., Seetharam, H. C., Kuhn, W. G., and Bright, J. T., "Aerodynamics of Spoiler Control Devices," AIAA Paper 79-1873, 1979.

${ }^{6}$ Wentz, Jr., W., Ostowari, C., and Seetharam, H., "Effects of Design Variables on Spoiler Control Effectiveness, Hinge Moments and Wake Turbulence," AIAA Paper 81-0072, 1981.

${ }^{7}$ Ok, H. and Eberhardt, D., "Calculation of the Flowfield Around an Airfoil with Spoiler," AIAA Paper 93-0527, 1993.

${ }^{8}$ Choi, S.-W. and Chang, K.-S., "Navier-Stokes Computations of a Rapidly Deploying Spoiler," AIAA Journal of Aircraft, Vol. 37, No. 4, pp. 655-661, 2000.

${ }^{9}$ Choi, S.-W., Chang, K.-S., and Ok, H., "Parametric Study of Transient Spoiler Aerodynamics with Two-Equation Turbulence Models," AIAA Journal of Aircraft, Vol. 38, No. 5, pp. 888-894, 2001.

${ }^{10}$ Yeung, W., Xu, C., and Gu, W., "Reduction of Transient Adverse Effects of Spoilers," AIAA Journal of Aircraft, Vol. 34, No. 4, pp. 479-484, 1997.

${ }^{11}$ Fillola, G., Pape, M.-C. L., and Montagnac, M., "Numerical Simulations Around Wing Control Surfaces," Paper, International Congress of The Aeronautical Sciences (ICAS), August/September 2004.

${ }^{12}$ Fillola, G., Carrier, G., and Dor, J.-B., "Experimental Study and Numerical Simulation of Flow Around Wing Control Surface," Paper, International Congress of The Aeronautical Sciences (ICAS), September 2006.

${ }^{13}$ Hantrais-Gervois, J.-L., Lepage, A., Ternoy, F., Carraz, G., and Jeanfaivre, G., "Assessment of Numerical Tools to Predict Control Surface Effectiveness," AIAA Paper 2010-4816, 2010.

${ }^{14}$ Jung, U. and Breitsamter, C., "Aerodynamic Wake Investigations of High-Lift Transport Aircraft With Deployed Spoilers," Paper, International Congress of The Aeronautical Sciences (ICAS), September 2010.

${ }^{15}$ Filippone, A., "Steep-Descent Maneuver of Transport Aircraft," AIAA Journal of Aircraft, Vol. 44, No. 5, pp. 1727-1739, 2007.

${ }^{16}$ Jung, U. and Breitsamter, C., "Aerodynamics of Multifunctional Transport Aircraft Devices," AIAA Paper 2010-4949, 2010.

${ }^{17}$ E.F.Sheta and Huttsell, L., "Numerical Analysis of F/A-18 Vertical Tail Buffeting," AIAA Paper 2001-1664, 2001.

${ }^{18}$ Brunet, V., "Computational Study of Buffet Phenomenon with Unsteady RANS Equations," AIAA Paper 2003-3679, 2003.

${ }^{19}$ Whitney, M. J., Seitz, T. J., and Blades, E. L., "Low-Speed-Stall Tail Buffet Loads Estimation Using Unsteady CFD," Paper 2009-127, International Forum on Aeroelasticity and Structural Dynamics (IFASD), June 2009.

${ }^{20}$ Hübner, A., "Experimental and numerical investigations of unsteady aerodynamic derivatives for transport aircraft configurations," AIAA Paper 2007-1076, 2007.

${ }^{21}$ Leatham, M., Stokes, S., Shaw, J., Cooper, J., Appa, J., and Blaylock, T., "Automatic Mesh Generation For RapidResponse Navier-Stokes Calculations," AIAA Paper 2000-2247, 2000.

${ }^{22}$ Martineau, D., Stokes, S., Munday, S., Jackson, A., Gribben, B., and Verhoeven, N., "Anisotropic Hybrid Mesh Generation for Industrial RANS Applications," AIAA Paper 2006-534, 2006.

${ }^{23}$ Brodersen, O., Crippa, S., Eisfeld, B., Keye, S., and Geisbauer, S., "DLR Results from the Fourth AIAA CFD Drag Prediction Workshop," AIAA Paper 2010-4223, 2010.

${ }^{24}$ Crippa, S., "Application of Novel Hybrid Mesh Generation Methodologies for Improved Unstructured CFD Simulations," AIAA Paper 2010-4672, 2010.

${ }^{25}$ Alrutz, T. and Rütten, M., "Investigation of Vortex Breakdown over a Pitching Delta Wing applying the DLR TAU-Code with Full, Automatic Grid Adaptation," AIAA Paper 2005-5162, 2005.

${ }^{26}$ Widhalm, M., Schütte, A., Alrutz, T., and Orlt, M., "Improvement of the Automatic Grid Adaptation for Vortex Dominated Flows using Advanced Vortex Indicators with the DLR-TAU code," New Results in Numerical and Experimental Fluid Mechanics VI, edited by C. Tropea, S. Jakirlic, and H. Heinemann, Vol. 96 of Notes on Numerical Fluid Mechanics and Multidisciplinary Design, Springer, Heidelberg, 2007, pp. 186-193.

${ }^{27}$ Gerhold, T., "Overview of the Hybrid RANS Code TAU," MEGAFLOW - Numerical Flow Simulation for Aircraft Design, edited by N. Kroll and J. Fassbender, Vol. 89 of Notes on Numerical Fluid Mechanics and Multidisciplinary Design, Springer, 2005, pp. 81-92.

${ }^{28}$ Schwamborn, D., Gerhold, T., and Heinrich, R., "The DLR TAU-Code: Recent Applications in Research and Industry," In Proceedings of the European Conference on Computational Fluid Dynamics, ECCOMAS, CFD 2006, edited by P. Wesseling, E. Oñate, and J. Périaux, The Netherlands, 2006.

${ }^{29}$ Jameson, A., Schmidt, W., and Turkel, E., "Numerical Solution of the Euler Equations by Finite Volume Methods using Runge-Kutta Time Stepping Schemes," AIAA Paper 81-1259, 1981.

${ }^{30}$ Spalart, P. and Allmaras, S., "A One-Equation Turbulence Model for Aerodynamic Flows," AIAA Paper 1992-439, 1992.

${ }^{31}$ Menter, F. R., "Two-Equation Eddy-Viscosity Turbulence Models for Engineering Applications," AIAA Journal, Vol. 32, No. 8, 1994, pp. 1598-1605. 
${ }^{32}$ Eisfeld, B., "Numerical Simulation of Aerodynamic Problems with the SSG/LRR- $\omega$ Reynolds Stress Turbulence Model Using the Unstructured TAU Code," New Results in Numerical and Experimental Fluid Mechanics VI, edited by C. Tropea, S. Jakirlic, and H. Heinemann, Vol. 96 of Notes on Numerical Fluid Mechanics and Multidisciplinary Design, Springer, Heidelberg, 2007, pp. 356-363.

${ }^{33}$ Brodersen, O., Eisfeld, B., Raddatz, J., and Frohnapfel, P., "DLR Results from the Third AIAA CFD Drag Prediction Workshop," AIAA Journal of Aircraft, Vol. 45, No. 3, pp. 823-836, 2008.

${ }^{34}$ Mertins, R., Elsholz, E., Barakat, S., and Colak, B., "3D viscous flow analysis - Dreidimensionale reibungsfreie Strömungsuntersuchungen an Flügel-Rumpf-Querruder-Spoiler Konfigurationen," Vol. 9 of Aerospace Science and Technology, Elsevier, 2005, pp. 476-484. 